\title{
Segregation in diffusion-limited multispecies pair annihilation
}

\author{
H.J. Hilhorst $\dagger$, O. Deloubrièreł, M.J. Washenberger $\$$, and \\ U.C. Täuber \\ † Laboratoire de Physique Théorique, Bâtiment 210 Université de Paris-Sud, F-91405 \\ Orsay cedex, France \\ $\ddagger$ Department of Physics, Virginia Polytechnic Institute and State University, \\ Blacksburg, Virginia, 24060, USA
}

\begin{abstract}
The kinetics of the $q$ species pair annihilation reaction $\left(A_{i}+A_{j} \rightarrow \varnothing\right.$ for $1 \leq i<j \leq q)$ in $d$ dimensions is studied by means of analytical considerations and Monte Carlo simulations. In the long-time regime the total particle density decays as $\rho(t) \sim t^{-\alpha}$. For $d=1$ the system segregates into single species domains, yielding a different value of $\alpha$ for each $q$; for a simplified version of the model in one dimension we derive $\alpha(q)=(q-1) /(2 q)$. Within mean-field theory, applicable in $d \geq 2$, segregation occurs only for $q<1+(4 / d)$. The only physical realisation of this scenario is the twospecies process $(q=2)$ in $d=2$ and $d=3$, governed by an extra local conservation law. For $d \geq 2$ and $q \geq 1+(4 / d)$ the system remains disordered and its density is shown to decay universally with the mean-field power law $(\alpha=1)$ that also characterises the single-species annihilation process $A+A \rightarrow \varnothing$.

PACS numbers: 05.40.-a, 82.20.-w
\end{abstract}

\section{Introduction}

\subsection{Motivation}

Thermal equilibrium is a very special situation and nature provides us with numerous examples of systems that cannot be described by equilibrium statistical thermodynamics. Investigation of simple models is necessary to understand the various dynamical phenomena observed in out-of-equilibrium systems, such as collective behaviour, phase transitions, or self-ordering [1, 2]. Among these models reactiondiffusion processes play a paradigmatic role because of their simple definition on the microscopic level and the complex phenomena they may exhibit on the macroscopic level $[3,4,5]$. Reaction-diffusion processes may describe not only chemical reactions $[6,7]$, but also e.g. phase transitions in ionic conductors [8], epidemic spreading [9], or forest fires [10]. The study of such simply defined models is justified by the fact that they entail macroscopic phenomena that are universal, i.e. that in the regime of asymptotically long times and large distances do not depend on the details of microscopic dynamics (for recent reviews, see Refs. [11, 12]). 


\subsection{Annihilation models}

The simplest nontrivial diffusion-limited reaction is the single-species pair annihilation process where random walkers (particles of type $A$ ) annihilate each other according to $A+A \rightarrow \varnothing$ when they meet. Here $\varnothing$ stands for an inert species that plays no further role in the dynamics. The diffusion-limited coagulation process $A+A \rightarrow A$ displays the same asymptotic behaviour [13]. An experimental realisation is the diffusion and annihilation of photo-generated excitons on tetramethylammonium manganese trichloride [14]. In the long-time regime, for systems with spatial dimension $d$, the particle density decays as $\rho(t) \sim t^{-1}$ for $d>2$, which is the mean-field result, whereas $\rho(t) \sim t^{-d / 2}$ for $d<2$. Exactly at the upper critical dimension $d_{c}=2$ one finds logarithmic corrections $\rho(t) \sim t^{-1} \ln t$

These asymptotic power laws were first obtained by means of exact calculations for specific models [15]. Their universality is established through a mapping of the corresponding classical master equations onto a stochastic field theory [16, 17, 18], and its subsequent analysis by means of the dynamical renormalisation group (RG) $[13,19]$. In the field theory language, the simple annihilation and coagulation processes are characterised by the absence of any propagator renormalisation, whence the relevant length scale is the diffusion length $\ell(t) \sim t^{1 / 2}$. The reaction rate enters as a nonlinear vertex, whose perturbative renormalisation can be summed to all orders by means of a Bethe-Salpeter equation. At long length and time scales, the renormalised reaction rate approaches a universal RG fixed point for $d<2$. The solution of the Callan-Symanzik equation for the particle density then yields the aforementioned results [19].

The two-species annihilation process $A+B \rightarrow \varnothing$ (with no reactions occurring between alike particle types) exhibits interesting new phenomena, especially for $d<4$ $[4,5,20]$. For a random initial distribution of $A$ and $B$ particles with equal densities $\rho_{A}(0)=\rho_{B}(0)$, in the course of time segregation into monospecies domains emerges. The average domain size is given by the typical diffusion length, and hence grows as $\ell(t) \sim t^{1 / 2}$. The reactions happen only in narrow zones between these domains. As a consequence the total particle density decays more slowly than in the single-species process: Whereas the homogeneous mean-field rate equation solution $\rho(t) \sim t^{-1}$ holds for $d \geq 4$, one has $\rho(t) \sim t^{-d / 4}$ for $d<4$. The reaction zone width $\ell_{\text {int }}(t)$ also tends to infinity according to a power law, $\ell_{\text {int }}(t) \sim t^{\lambda_{r}}$, with, in particular, $\lambda_{r}=3 / 8$ in $d=1$ [21]. The renormalisation group analysis for this two-species process establishes that the upper critical dimension in the RG sense is still $d_{c}=2$, i.e., mean-field rate equations for the local particle densities augmented with diffusion to account for spatial variations provide an apt description for $d>2[20]$. Thus, the borderline dimension for segregation $d_{\text {seg }}=4$ is located inside the realm of validity of mean-field theory.

For unequal initial densities of the $A$ and $B$ species $[22,23]$, the final state will be characterised by a nonzero density of the majority species. Above $d_{c}=2$ the density $\rho_{\min }$ of the minority species decays exponentially. For $d \leq d_{c}$ this decay becomes a stretched exponential with $\ln \rho_{\min } \sim-t / \ln t$ for $d=2$ and $\ln \rho_{\min } \sim-t^{d / 2}$ for $d<2$. 
It is worth mentioning that certain special initial conditions with long-range correlations are capable of inhibiting segregation. As an example, consider an alternating alignment of particles ...ABABAB ... in one dimension, and reactions with infinite rate. Then any encounter will involve particles of distinct species, leading to their annihilation. Yet removing any neighbouring $A B$ pair will conserve the alternating alignment. Since alike particles can therefore never meet, this process is in fact equivalent to the single-species reaction $A+A \rightarrow \varnothing$. Segregation is impossible, and the total density decays as $\sim t^{-1 / 2}[24]$.

It is now natural to view the two above models as special cases of a Multispecies Annihilation Model (MAM) $[25,26,27]$ in which $q$ different species $A_{1}, A_{2}, \ldots, A_{q}$ perform random walks on a lattice and, when meeting on the same lattice site, may react according to

$$
A_{i}+A_{j} \rightarrow \varnothing, \quad i \neq j .
$$

We will consider that the hopping rates and the reaction rates are species-independent (i.e., we assume uniform diffusion constants $D_{i}=D$ and reaction rates $\lambda_{i j}=\lambda$ ). For equal initial densities $\rho_{i}(0)=\rho(0) / q$ one expects a power law decay

$$
\rho(t) \sim t^{-\alpha},
$$

with a $q$ - and $d$-dependent exponent that we denote as $\alpha(q, d)$, or, when we are interested only in its $q$ dependence, as $\alpha(q)$. In this paper we shall investigate under which conditions the MAM may exhibit species segregation, and we will determine the value of the exponent $\alpha$ as function of $q$ and $d$. Thus we fully characterise the longtime behaviour of a multicomponent reaction-diffusion system for arbitrary number of species, and elucidate the physics behind the different asymptotic scaling laws. Similar to other multispecies systems with hard-core constraints [29], the MAM process turns out to display very special features in one dimension, owing to the special topological limitations of hopping on chains.

One special limit of the MAM may be analysed immediately. For an infinite number of species, $q=\infty$, the probability for two particles of the same type to meet is zero. Therefore all encounters lead with rate $\lambda$ to a reaction. Distinguishing different particle species then loses its meaning, whence for $q=\infty$ we recover again the single-species pair process $A+A \rightarrow \varnothing$ in $d$ dimensions [25].

We remark that novel behaviour can be expected generically only in the highly symmetric case with uniform reaction rates and equal initial densities. In any other situation, one would expect that at long times only the least reactive and/or most populous species will survive. Once the minority particle species have disappeared, one should expect the process to be described by the $A+B \rightarrow \varnothing$ reaction with unequal particle densities [26].

\subsection{Results}

We shall present numerical and analytical results concerning the asymptotic decay of the total particle density $\rho(t)$ of the $q$-species MAM in $d$ dimensions. There are two physical 
effects that invalidate the homogeneous mean-field rate equation predictions in low dimensions, namely reaction rate renormalisation and potentially species segregation. Associated with these are two borderline dimensions. First, below the upper critical dimension $d_{c}=2$ (independent of $q$ ) fluctuations in the dynamics are relevant in the $\mathrm{RG}$ sense, renormalising the effective reaction rate $\lambda$. Second, within the mean-field approximation, below a $q$-dependent segregation dimension $d_{\text {seg }}$ given by

$$
d_{\mathrm{seg}}(q)=\frac{4}{q-1}
$$

the system splits up into monospecies domains. The behaviour predicted by the homogeneous mean-field rate equations (that do not allow for segregation) is observed only for $d>\max \left(d_{c}, d_{\text {seg }}\right)$.

The full picture of how the $q$-species MAM behaves in $d$ dimensions then emerges as follows:

- For $d=1$ the special topological constraints of a linear chain produce segregation with a nonuniversal value of the decay exponent that we determine $[26,27]$ to be

$$
\alpha(q, 1)=\frac{q-1}{2 q}, \quad d=1 .
$$

This expression of course reproduces the known one-dimensional exponents $\alpha(2,1)=1 / 4$ and $\alpha(\infty, 1)=1 / 2$.

- For $d \geq 2$ and $q=2$ an extra conservation law in the microscopic dynamics causes segregation and

$$
\alpha(2, d)=\frac{d}{4}, \quad 2 \leq d \leq d_{\mathrm{seg}}(2)=4
$$

Note that when $d=1$ is substituted in Eq. (5), the result coincides with $\alpha(2,1)$ as given by Eq. (4). The local conservation law for the particle density difference in two-species annihilation $A+B \rightarrow \varnothing[5]$ renders the case $q=2$ quite special.

- If one is willing to analytically continue the results to noninteger $q$, then Eq. (5) is a special case of

$$
\alpha(q, d)=\frac{(q-1) d}{4}, \quad 2 \leq d \leq d_{\mathrm{seg}}(q), \quad 1<q<3 .
$$

For all $(q, d)$ in this range there occurs segregation, although for $q \neq 2$ it cannot be traced back to a conservation law.

- For $d \geq 2$ and $q=3,4, \ldots, \infty$ there is no segregation. The density decay therefore follows a universal (mean-field) power law, independent of the number of species:

$$
\alpha(q, d)=1, \quad d \geq 2, \quad q \geq 3 .
$$

In the borderline case $d=2$ the power law defined by Eq. (7) is accompanied by a logarithmic correction, $\rho(t) \sim t^{-1} \ln t$, precisely as for $A+A \rightarrow \varnothing$.

In one dimension, when segregation occurs, domains of identical particles are separated by reaction zones where the annihilation reactions takes place. Let $\ell_{\text {int }}$ be 
the typical distance between closest-neighbour pairs $A_{i} A_{j}$ in a reaction zone. We will now assume an infinite reaction rate, so that there is one such pair per zone and $\ell_{\text {int }}$ is also the zone width. The temporal growth of $\ell_{\text {int }}$ may be analysed as follows. The density of zones is equal to $1 / \ell(t)$. Hence we may write the rate of decrease of the total density as

$$
\frac{\mathrm{d} \rho(t)}{\mathrm{d} t}=-\frac{\kappa(t)}{\ell(t)},
$$

where $\kappa(t)$ is the typical number of annihilations per unit time in a reaction zone. This relation, which may be taken as the definition of $\kappa(t)$, was proposed and exploited by Leyvraz and Redner [21]. The asymptotic time dependence of $\kappa(t)$ is connected by Eq. (8) to those of $\rho(t)$ and $\ell(t)$. The time needed for the pair $A_{i} A_{j}$ to annihilate is $\sim \ell_{\text {int }}^{2}$, so the change of particle density per unit time is $\mathrm{d} \rho / \mathrm{d} t \sim 1 /\left(\ell \ell_{\text {int }}^{2}\right)$. If we now set $\rho(t) \sim t^{-\alpha}$ and $\ell_{\text {int }} \sim t^{\lambda_{r}}$, with $\ell \sim t^{1 / 2}$, then Eq. (8) yields the scaling relation [26, 27]

$$
\lambda_{r}(q)=\frac{1}{2}\left[\alpha(q)+\frac{1}{2}\right]=\frac{2 q-1}{4 q}, \quad d=1 .
$$

For the two-species model one recovers $\ell_{\text {int }} \sim t^{3 / 8}[21]$.

This paper is organised as follows. In Sec. 2 we recall the known methods and results for the $q=2$ and $q=\infty$ cases in a field-theoretic perspective. We then extend these arguments to any value of $q$. We show why it is difficult in $d<2$ to obtain results for general $q$ by renormalisation methods. In Sec. 3 we change our approach and consider dimension $d=1$ specifically. We introduce a simplified version of the MAM, to be referred to as SMAM, in which the stochastic dynamics is replaced by a deterministic algorithm. This SMAM is exactly solvable and produces the asymptotic density exponent (4). We argue that this result is valid also for the original stochastic MAM. Our analytical findings are supported by our own Monte Carlo simulations, presented in Sec. 4, as well as by recent data by Zhong et al. [27]. We show that great caution must be exercised when measuring decay exponents, since crossover regimes can be long (and depend on the ratio of the reaction rate and the diffusion constant). Sec. 5 contains some concluding remarks. Appendix A details the solution of the mean-field rate equations. In Appendix B we point out the topological specificities of the one-dimensional case by mapping the model onto an anisotropic antiferromagnetic Heisenberg spin chain.

\section{Analytical considerations}

\subsection{Mean-field theory}

The kinetics of chemical reactions may in first approximation be investigated by mean-field theory. The mean-field rate equations neglect any spatial variations of the particle densities and thus cannot capture reaction-induced noise and diffusioninduced correlations. In low dimensions, however, these effects may govern the long-time behaviour of the reaction kinetics, invalidating the mean-field approximation. 
For the MAM, the mean-field rate equations (with a uniform reaction rate absorbed into the time scale) read

$$
\frac{\mathrm{d} \rho_{i}(t)}{\mathrm{d} t}=-\sum_{\substack{j=1 \\ j \neq i}}^{q} \rho_{i} \rho_{j}, \quad i=1,2, \ldots, q .
$$

For equal initial densities $\rho_{i}(0)=\rho^{*}$, Eqs. (10) are easily solved to give $\rho(t)=$ $r h o^{*} /\left[1+(q-1) \rho^{*} t\right]$. Above some critical dimension, $d>\max \left(d_{c}, d_{\mathrm{seg}}\right)$ therefore expect the MAM decay exponent $\alpha$ to take on the mean-field value $\alpha(q, d)=1$.

It is of interest to consider Eqs. (10) when the permutation symmetry of the species is broken by the initial conditions, i.e., when the $\rho_{i}(0)$ are not all equal. For $k=1, \ldots, p$, let there be initial values $\rho_{k}(0)=\rho_{k}^{*}$ having multiplicity $n_{k}$, where $\sum_{k=1}^{p} n_{k}=q$. We then arrive at a set of $p>1$ independent mean-field equations which, after renumbering the species and absorbing the rate $\lambda$ into the time scale, may be written as

$$
\frac{\mathrm{d} \rho_{k}(t)}{\mathrm{d} t}=-\rho_{k}\left(\sum_{\ell=1}^{p} n_{\ell} \rho_{\ell}-\rho_{k}\right), \quad k=1, \ldots, p,
$$

with the initial conditions now all distinct and ordered such that

$$
0<\rho_{1}^{*}<\rho_{2}^{*}<\ldots<\rho_{p}^{*} .
$$

The study of this nonlinear system of equations was initiated by Ben-Avraham and Redner [25]. In Appendix A we complete their analysis and determine the full analytic solution of the equations (11) and (12) for general $p$ and $\left\{n_{\ell}\right\}$. The asymptotic large time behaviour may be summarised as follows.

- For $n_{p}>1$ the density $\rho_{p}$ of the most abundant species is $n_{p}$-fold degenerate. It decays to zero as $t^{-1}$. The densities of the other species tend to zero with a faster power common to all of them, viz. as $t^{-1-1 /\left(n_{p}-1\right)}$.

- For $n_{p}=1$ the density of the most abundant species, $\rho_{p}$, is nondegenerate. It tends exponentially to a constant $\rho_{p}^{*} A^{*}$ with $A^{*}$ given by

$$
A^{*}=\prod_{\ell=1}^{p-1}\left(1-\rho_{\ell}^{*} / \rho_{p}^{*}\right)^{n_{\ell}}
$$

The densities of all other species tend exponentially to zero for asymptotically large times on the common time scale $\tau=1 / \rho_{p}^{*} A^{*}$.

\subsection{A Ginzburg-type criterion for the segregation dimension $d_{\mathrm{seg}}$}

The homogeneous mean-field approximation of the preceding subsection 2.1 is based on the hypothesis of there being no particle species segregation. Still within the framework of mean-field theory, but allowing now for particle density inhomogeneities, we will provide a criterion for the appearance of segregation. To this end, we investigate local deviations from the global density averages. When it will appear that these deviations are of the same order as the averages, one must conclude that the no-segregation hypothesis becomes invalidated. 
Let there be an infinite system with equal initial densities $\rho^{*}$ for all $q$ species. We will consider a finite subvolume $L^{d}$ of this system. Within such a volume the initial densities, due to random fluctuations, will be

$$
\rho_{i L}(0)=\rho^{*}+c_{i} \sqrt{\rho^{*}} L^{-d / 2}, \quad i=1,2, \ldots, q,
$$

where the $c_{i}$ are random constants of order unity with zero average. For times $t$ small compared to the diffusion time across the volume, $t \lesssim t_{L} \sim L^{2} / D$, this volume may be considered as isolated from the rest of the system. We now assume the mean-field approximation to be valid. Generically all $c_{i}$ will be different. The $\rho_{i L}(t)$ then evolve in time according to Eq. (11) with all $n_{k}=1$ and initial conditions given by Eq. (14). Let $c_{q}$ be the largest one of the random constants. Hence $\rho_{i L}(t)$ will tend to zero for all $i=1, \ldots, q-1$, whereas $\rho_{q L}(t)$ will approach the limit $\rho_{q L}(\infty)=\rho^{*} A_{L}^{*}$, with, according to Eq. (13),

$$
\begin{aligned}
A_{L}^{*} & =\prod_{i=1}^{q-1}\left[1-\rho_{i L}(0) / \rho_{q L}(0)\right] \\
& \simeq\left[\prod_{i=1}^{q-1}\left(c_{q}-c_{i}\right)\right]\left(\rho^{*}\right)^{-(q-1) / 2} L^{-(q-1) d / 2} \simeq C L^{-(q-1) d / 2} .
\end{aligned}
$$

Therefore in an isolated volume $L^{d}$ only a single species will be left over, with density $\sim L^{-(q-1) d / 2}$. The time scale for the approach of this limiting value is $\tau_{L}=1 / \rho^{*} A_{L}^{*} \sim$ $L^{(q-1) d / 2}$. This time scale is well within the time that the volume stays isolated at the condition that $\tau_{L} \lesssim t_{L}$, i.e., if $(q-1) d / 2<2$ or equivalently $d<d_{\text {seg }}$, where $d_{\mathrm{seg}}=4 /(q-1)$ is the critical segregation dimension announced in Eq. (3). Hence for $d>d_{\text {seg }}$ diffusion prevents segregation, but for $d<d_{\text {seg }}$ the reactions occur so fast that they segregate the system into single-species domains.

Segregation decelerates the decay of the total density. To determine precisely how that happens, we observe that at any time $t$ the total density $\rho(t)$ is equal to the limit density $\rho^{*} A_{L(t)}^{*}$ in a volume of corresponding size, $L(t)^{d}$, where $L(t) \sim(t / D)^{1 / 2}$. Combining this with Eq. (15) we find that asymptotically for $t \rightarrow \infty \rho(t) \sim t^{-(q-1) d / 4}$, valid when segregation occurs under mean-field conditions. Hence in this situation we obtain the decay exponent $\alpha(q, d)=(q-1) d / 4$ as given in Eq. (6).

As we will see in subsection 2.3, the mean-field approximation, with or without segregation, is valid only for $d>d_{c}$ (and marginally valid in $d=d_{c}$ ), where $d_{c}=2$ is the $q$-independent upper critical dimension. It follows that in the mean-field part of the $(q, d)$ plane the only physical systems (i.e., with integer $q$ and $d$ ) showing segregation are the two-species $(q=2)$ annihilation processes in $d=2$ and $d=3$ dimensions. We cannot exclude, however, that noninteger values of $q$ might someday be given a physical interpretation as well.

It so happens that the $q=2$ system satisfies a microscopic conservation law: The difference $N_{A}-N_{B}$ between the particle numbers of the two species $A$ and $B$ is strictly conserved in each reaction. This law may therefore be viewed as being at the origin 
of the segregation effect. In any case, for $q \geq 3$ and $d>2$ we conclude that the homogeneous mean-field results of subsection 2.1 apply.

It is interesting to remark that the derivation of our expression (3) may also be based on Ref. [25]. Here, the authors set $\rho_{i}(t)=\rho(t)+c_{i}(t) \sqrt{\rho^{*}} L^{-d / 2}$, i.e., the time-dependent generalization of our Eq. (14), and by expanding the master equation they show that the mean-square fluctuations $c(t)^{2} \equiv\left\langle\left(c_{i}(t)-c_{j}(t)\right)^{2}\right\rangle$ obey an ordinary differential equation in time [their Eq. (29)]. The solution of that equation, which was also examined very recently in Ref. [28], shows that for $1<q<3$ the typical fluctuation $c(t) \sqrt{\rho^{*}} L^{-d / 2}$ becomes of the order of the density $\rho(t)$ on the same time scale $\tau_{L}$ that appeared above in our study of the nonlinear solution; whence we obtain the same result for $d_{\text {seg. }}$.

\subsection{Renormalisation group arguments}

In dimension $d \leq d_{c}=2$ the considerations of the two preceding subsections 2.1 and 2.2 are no longer valid. Fluctuations in these lower dimensions, where the reactions become effectively diffusion-limited, need to be taken into account beyond the Gaussian approximation. A systematic treatment of fluctuations in reaction-diffusion problems starts from the associated classical master equation, utilises a standard representation in terms of bosonic creation and annihilation operators, and therefrom builds a field theory action by means of coherent state path integrals, see, e.g., Refs. [16, 17, 13, 19]. This action is then further analysed using dynamic renormalisation group ( $R G$ ) methods, and the ensuing scaling laws can be extracted from the Callan-Symanzik flow equation.

For the $q$-species MAM the ensemble average at time $t$ of any observable $F$ can be expressed in terms of functional integrals over $2 q$ fields $\left\{\phi_{i}\right\}_{i=1}^{q}$ and $\left\{\hat{\phi}_{i}\right\}_{i=1}^{q}$ as

$$
\langle F\rangle_{t}=\frac{\int \prod_{i=1}^{q} \mathcal{D} \hat{\phi}_{i} \mathcal{D} \phi_{i} \bar{F}\left(\left\{\hat{\phi}_{i}\right\},\left\{\phi_{i}\right\}\right) \mathrm{e}^{-S\left[\left\{\hat{\phi}_{i}, \phi_{i}\right\}\right]}}{\int \prod_{i=1}^{q} \mathcal{D} \hat{\phi}_{i} \mathcal{D} \phi_{i} \mathrm{e}^{-S\left[\left\{\hat{\phi}_{i}, \phi_{i}\right\}\right]}}
$$

where the functional $\bar{F}\left(\left\{\hat{\phi}_{i}\right\},\left\{\phi_{i}\right\}\right)$ is uniquely determined by the observable $F$ (e.g., the total particle density is given by $\left.\sum_{i=1}^{q} \hat{\phi}_{i} \phi_{i}\right)$. The statistical weight of any configuration is given by the action

$$
\begin{aligned}
\mathcal{S}\left[\left\{\hat{\phi}_{i}, \phi_{i}\right\}\right]=\sum_{i=1}^{q} \int \mathrm{d}^{d} r & \left\{\int_{0}^{\infty} \mathrm{d} t\left[\hat{\phi}_{i}\left(\partial_{t}-D \nabla^{2}\right) \phi_{i}-\lambda \sum_{\substack{j=1 \\
j \neq i}}^{q}\left(1-\hat{\phi}_{i} \hat{\phi}_{j}\right) \phi_{i} \phi_{j}\right]\right. \\
& \left.-\rho_{i}(t=0) \hat{\phi}_{i}(t=0)\right\}
\end{aligned}
$$

The bilinear part of this action defines $q$ diffusion propagators, whereas the reactions introduce $q(q-1) / 2$ two- and four-point annihilation vertices, as depicted in Fig. 1 (a). Here, the bare coupling $\lambda$ represents the continuum version of the uniform annihilation rate of the discrete model. Straightforward power counting indicates that fluctuations become relevant in $d<2$ dimensions, for any value of $q$. Hence we identify $d_{c}=2$ as the upper critical dimension in the RG sense. Thus we need to distinguish three cases:

(i) For dimensions $d>2$ the fluctuation contributions are convergent in the infrared, and standard perturbation theory is applicable. Therefore the mean-field equations (10), 
(a)

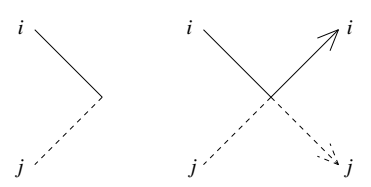

(b)

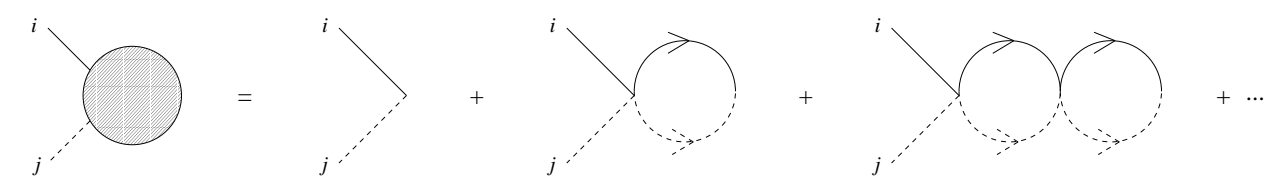

(c)

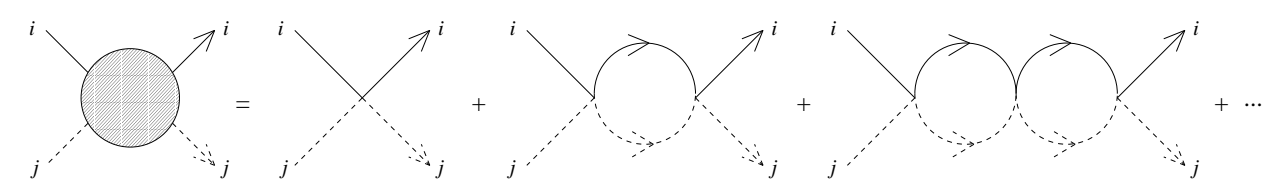

Figure 1. (a) The propagators and vertices of the bosonic field theory. (b,c) Renormalisation of the two- and four-point vertices, respectively.

augmented with diffusive terms, should yield the correct asymptotic behaviour, whether or not species segregation appears. For values $q \geq 3$, the critical segregation dimension is $d_{\text {seg }} \leq 2$, so segregation will not occur. Thus the long-time decay of the particle densities will follow the homogeneous mean-field results described in subsection 2.1. Specifically, for equal initial densities $\rho_{i}(0)=\rho(0) / q$ the value of the decay exponent is universally $\alpha=1$, independent of $q$ and $d$. For the two-species model $(q=2)$, subsection 2.2 predicts that segregation happens in dimensions $d<4$. As explained in more detail in subsection 2.4 below, this is in fact associated with the local conservation of the particle density difference $\rho_{1}-\rho_{2}$, which is a very special feature of the two-species annihilation model. For equal particle densities this leads to the slower decay $\rho(t) \sim t^{-d / 4}[4,5,20]$.

(ii) At the critical dimension $d_{c}=2$ (and below), the full renormalisation group machinery is required to further analyse the action (17). As illustrated in Fig. 1(b) and (c), the Feynman diagrams associated with the perturbative renormalisation of the annihilation rate assume identical structures for all $q$. Moreover, the resulting RG flow behaves as for the single-species annihilation process $A+A \rightarrow \varnothing$ (see Refs. [13, 20] for the two-species case). In two dimensions, the associated running renormalised coupling tends to zero logarithmically for large length- or timescales. Consequently, only the treelevel Feynman diagrams involving the initial state enter the computation of the average particle densities, $c f$. subsection 2.4. We may then immediately conclude that for $q \geq 3$, the mean-field results of subsection 2.1 acquire straightforward logarithmic corrections, independent of the value of $q$ : If the density of the most abundant particle species is nondegenerate $\left(n_{p}=1\right)$, it will approach a constant at long times. The approach to this asymptotic value, as well as the vanishing of all other species, is characterised by a stretched exponential $\ln \left[\rho_{i}(t)-\rho_{i}(\infty)\right] \sim-t / \ln t$. In the degenerate case $\left(n_{p}>1\right)$, which includes equal initial densities $\rho_{i}(0)=\rho(0) / q$ as a special situation $\left(n_{p}=q\right)$, one obtains $\rho(t) \sim t^{-1} \ln t$. Only the two-species MAM with equal initial densities 
has distinct features, with $\rho(t) \sim t^{-1 / 2}$, owing to the local conservation law and the ensuing particle segregation (see subsection 2.4). In Sec. 4, we shall support the above predictions by means of Monte Carlo simulations in two and three dimensions.

(iii) In dimensions $d<2$, as explained above, the 'bulk' renormalisation for the reaction rate proceeds precisely as for the single-species annihilation model, see Fig. 1(b) and (c). However, this does not imply that the ensuing asymptotic density decay power law holds as well. For now, in order to compute observables such as the averages $\left\langle\rho_{i}(t)\right\rangle$, one must sum all Feynman diagrams that contain contributions from relevant operators on the initial time sheet $(t=0)$. For the $q$-species MAM, this turns out to be a formidable task. Before we discuss this issue further, let us recall the situation in the two-species annihilation model.

\subsection{Effects of the local conservation law for $q=2$}

The renormalisation of the annihilation rate $\lambda$ that captures the emergence of particle anticorrelations and depletion zones remains present (absent) in dimensions $d \leq d_{c}=2$ $(d>2)$ and unchanged even if there exists an additional microscopic conservation law. Yet physical effects associated with the latter, such as particle segregation, may crucially affect the system's leading asymptotic behaviour. Such phenomena, though encoded in the initial state, viz. the $t=0$ time sheet in the action (17), are in general more difficult to analyse in field theory than 'bulk' renormalisations.

We illustrate this with a brief review of the situation for $q=2$, following Ref. [20]. The two-species pair annihilation process is very special owing to a local conservation law (not valid for any other value of $q$ ), namely for the particle density difference $\rho_{1}(t)-\rho_{2}(t)=\rho_{1}(0)-\rho_{2}(0)[5]$. As a consequence, the initial state at $t=0$ propagates into the 'bulk' $(t>0)$ all the way to $t \rightarrow \infty$, seriously complicating the analytical approach. To determine the asymptotic behaviour of the density Lee and Cardy [20] used the following procedure. After shifting the response fields according to $\hat{\phi}_{i} \rightarrow \bar{\phi}_{i}=\hat{\phi}_{i}-1$, new fields $\psi=\left(\phi_{1}+\phi_{2}\right) / \sqrt{2}$ and $\xi=\left(\phi_{1}-\phi_{2}\right) / \sqrt{2}$ are introduced, along with analogous transformations for the response fields $\bar{\psi}$ and $\bar{\xi}$. To account for the effect of the initial state, specifically for equal initial densities, an effective action is built that contains all possible initial terms compatible with the symmetries of the problem. These can generically be written as $\Delta^{(m, n)} \bar{\psi}^{m}(0) \bar{\xi}^{n}(0)$. The most relevant coupling responsible for the leading-order decay of the density turns out to be $\Delta=\Delta^{(0,2)}=-\Delta^{(2,0)}$, whose canonical scaling dimension is $4-d$.

For dimensions $d>4$ therefore, the initial state plays no crucial role, and the asymptotic behaviour is determined by the homogeneous mean-field rate equations. For $2<d \leq 4$, when the 'bulk' parameters in the model do not require any renormalisation, a simple effective theory can be constructed. Aside from the new term $\Delta \bar{\xi}^{2}(0)$, it resembles the mean-field rate equations, supplemented with diffusion terms. Since it is a locally conserved mode, the density difference $\xi(t)$ propagates diffusively, describing the growth of segregated regions in time. This already implies $\rho_{i}(t) \sim t^{-d / 4}$ as $t \rightarrow \infty$ 
for equal initial densities. The amplitude of this power law is determined by summing all tree-level Feynman diagrams that involve the initial parameters $\rho(0)$ and $\Delta$, with the ultimate result $[30,31]$

$$
\rho_{1,2}(t) \sim \sqrt{\frac{\rho(0)}{\pi}} \frac{1}{(8 \pi D t)^{d / 4}} \quad \text { as } t \rightarrow \infty, \quad 2<d \leq 4 .
$$

In lower dimensions $d \leq 2$, however, one needs to apply the full machinery of the renormalisation group in order to calculate the $\langle\psi\rangle$ by means of a perturbative expansion with respect to the parameter $\varepsilon=2-d$. Precisely as in the single-species pair annihilation process [19], the renormalised dimensionless counterpart of the reaction rate $\lambda$ flows towards a stable fixed point of order $\varepsilon$. Yet the calculation of observables in the framework of this $\varepsilon$ expansion still necessitates nonperturbative summations over all orders of the initial parameters $\rho(0)$ and $\Delta[20]$. Whereas this is a straightforward task for the single-species reaction, this goal has to date not been achieved beyond the leading order in $\varepsilon$ even for the two-species pair annihilation process. For $\varepsilon=0$, where the renormalised running coupling associated with the annihilation rate vanishes logarithmically, this suffices to establish the validity of the result (18) even at the upper critical dimension $d_{c}=2$. For $d<2$, though, the field-theoretic treatment in principle leaves three possibilities: Higher-order terms may have no crucial effect at all, maintaining the validity of Eq. (18); they may alter the amplitude of the asymptotic power law, but leave the exponent $\alpha(2, d)=d / 4$ intact; or they might even lead to a different asympotic algebraic behaviour. However, this last scenario is not supported by simulations in $d=1[5,22]$ and exact results on a specific variant of the two-species annihilation model [23].

The arguments of Ref. [20] summarised here show that in order to extract the value of $\alpha(q)$ in $d=1$ within a bosonic field theory, one must perform a sum over all diagrams that contain relevant initial state operators. Even for the two-species annihilation model this summation appears very difficult, if not impossible. We hence expect the analysis to be even more cumbersome for the $q$-species process. Yet the possibility of species segregation must be encoded precisely in these contributions from the initial state.

To explain the $q$-dependence of the asymptotic regime, one then needs a field theory representation where the special topology of the one-dimensional case becomes manifest. An example is provided by the theory of quantum spin chains. In Appendix B, we discuss how the annihilation model can be mapped onto an anisotropic antiferromagnetic Heisenberg spin chain. The appropriate continuum limit for the low-lying excitation yields generalised nonlinear sigma models with topological Chern-Simons terms. For the $q$-species MAM, the different topological sectors for each $q$ will become mixed through the annihilation processes. We conjecture that the presence of these topological terms, effective only near one dimension, must be responsible for the $q$-dependence of the density decay exponent in the asymptotic regime. Yet the ensuing formalism is not readily amenable to a direct calculation of $\alpha(q, 1)$ either. In view of these difficulties, we shall adopt a very different approach to the one-dimensional $q$-MAM in Sec. 3. In higher 
dimensions $d \geq 2$ the topological terms do not play any significant role, which supports that the RG analysis of section 2.3 predicts the correct MAM asymptotic behaviour.

\subsection{Role of special initial conditions}

Yet before proceeding, we note that special initial conditions can alter the generic asymptotic behaviour of the $q$-MAM. For instance, if the particles live on a onedimensional lattice, their respective rank can be labeled starting from a given origin. Since particles only vanish in pairs, the parity of each particle rank is a locally conserved quantity. This immediately implies that if one starts with a correlated configuration such as

$$
\ldots A_{q-1} A_{q} A_{1} A_{2} \ldots A_{q-1} A_{q} A_{1} A_{2} \ldots
$$

the parity of $q$ will dictate the long-time decay of the density. On the one hand, if $q$ is odd, two particles of the same species always have an even number of particles between each other. Hence these particles can annihilate by pairs and two particles of the same species can become nearest-neighbours after some annihilation reactions have occurred. Therefore the subtle initial correlations will be wiped out eventually and segregation becomes possible, leading to an asymptotic density decay as in the $q$-MAM (albeit following a potentially large crossover period). On the other hand, if $q$ is even, the particles lying between two identical particles cannot entirely annihilate each other. In that situation, particles of the same species never meet on adjacent lattice sites, species segregation cannot occur, and the density decays as in the single-species pair annihilation reaction $A+A \rightarrow \varnothing$, i.e., with $\alpha=1 / 2$.

\section{Simplified one-dimensional model}

\subsection{Simplified Mutual Annihilation Model (SMAM)}

In order to address the one-dimensional case, we now introduce a simplified version of the MAM, to be referred to as SMAM, which retains only the bare essentials of the original model. We begin by noting that the particle configuration may at any time be decomposed into a sequence of domains, each comprising only a single particle species, such that adjacent domains contain different species. Owing to the diffusive nature of the process the typical domain size increases as $\ell(t) \simeq(D t)^{1 / 2}$, as we shall confirm numerically in Sec. 4. Combining this with the expectation that $\rho(t) \sim(D t)^{-\alpha}$, we may express the average particle number $\bar{n}(t)=\ell(t) \rho(t)$ in a domain as

$$
\bar{n}(t) \sim(D t)^{\frac{1}{2}-\alpha} .
$$

Clearly, unbounded domain growth, hence species segregation, can only occur if $\alpha<1 / 2$. The emergence of segregation thus necessarily implies a slower density decay than predicted for the homogeneous system $(\alpha=1 / 2)$ in one dimension.

Inspired by Eq. (8) we now introduce the hypothesis that fluctuations in the effective

annihilation rate $\kappa(t)$, whether in the course of time or between different reaction zones, 
are not crucial and may be ignored because in one dimension the segregation effect will dominate at least the long-time dynamics. This leads us directly to the SMAM picture: The particle content of each domain, owing to the annihilation reactions taking place at both of its ends, decreases at the constant rate $2 \kappa(t)$. This picture is then completed by the rule for what happens when a domain disappears, i.e., gets emptied of all its particles: Then, either the left and right neighbouring domains have the same species of particles and fuse into a single new domain; or the neighbouring domains have different particle species and a new reaction zone appears between them. These two possibilities correspond to annihilation and coalescence of domain walls, respectively. We already note that it is precisely via these two different scenarios, whose respective probabilities depend on the overall number of species, that the decay exponent $\alpha(q)$ eventually becomes a function of $q$.

The following algorithm makes this explicit. We consider a one-dimensional lattice ('chain') whose sites, labeled by indices $i, j, \ldots$, represent the domains of the original MAM. Below we will indifferently refer to them as 'sites' or as 'domains'. Their initial number will be denoted by $N_{0}$. To start, we randomly select $N_{0}$ number variables $n_{10}, n_{20}, \ldots, n_{N_{0} 0}$, drawn independently from some probability distribution on the positive integers; these variables represent the initial numbers of particles in the domains. We then assign initially to each domain a particle type ('colour') in a random fashion, except for the restriction that neighbouring domains are not allowed the same colour. Yet note that the subsequent algorithm does not require that we assign these colours explicitly. The time evolution subsequent to this random initial condition proceeds via deterministic iterations. The $(k+1)$ th iteration changes the total number of sites from $N_{k}$ to $N_{k+1}$ and converts the set of values $\left\{n_{i k}\right\}_{i=1}^{N_{k}}$ to $\left\{n_{j, k+1}\right\}_{j=1}^{N_{k+1}}$. The following update consists of four steps:

(i) The values of all $n_{i k}$ are reduced by 1 .

(ii) All sites $i$ which after step (i) have $n_{i k}=0$ are eliminated from the lattice. The remaining sites are reconnected in such a way that their ordering along the chain remains unchanged.

(iii) Sites which as a result of step (ii) have become nearest-neighbour sites and which have the same colour, are fused into a single site of that colour; the number variables of the fusing sites are added together.

(iv) We write $N_{k+1}$ for the remaining number of sites, and relabel these sites by an index $j$ that runs from 1 to $N_{k+1}$ along the chain.

We began our investigation by actually running this algorithm on the computer, and found these simulations to converge rapidly and yield accurate exponent values. These advantages were however superseded by our discovery that the above algorithm is actually amenable to an asymptotically exact solution.

In order to keep track of the colours of the sites, in our computer implementations of this algorithm we also used a set of colour variables $\left\{\sigma_{i k}\right\}_{i=1}^{N_{k}}$, where $\sigma_{i k} \in\{1,2, \ldots, q\}$ represents the colour of the $i$ th site after the $k$ th iteration. In our discussion below of the 
analytic solution of the algorithm our dealing with these variables will be implicit. The sole quantity we need to know is the probability, to be denoted $\eta_{r}$, that two domains separated by $r$ other domains have the same colour, i.e., that $\sigma_{i k}=\sigma_{i+r+1, k}$. Obviously, $\eta_{0}=0$ and $\eta_{1}=1 /(q-1)$. Since the two variables $\sigma_{i k}$ and $\sigma_{i+r+1, k}$ can be equal only if $\sigma_{i k} \neq \sigma_{i+r, k}$, and in that case in fact coincide with probability $1 /(q-1)$, it follows that $\eta_{r}$ obeys the recursion relation $\eta_{r}=\left(1-\eta_{r-1}\right) /(q-1)$, whence

$$
\eta_{r}=\left[1-(1-q)^{-r}\right] / q, \quad r=0,1,2, \ldots .
$$

Thus the domain colours exhibit an oscillatory short-ranged correlation. It is easy to see that this correlation is in fact invariant under an iteration step.

After the $k$ th iteration let $\bar{n}_{k}=N_{k}^{-1} \sum_{i=1}^{N_{k}} n_{i k}$ denote the average number of particles per domain. Let furthermore $\rho_{k}$ denote the particle density and $t_{k}$ the physical time after $k$ iterations. In terms of the data produced by the algorithm, these two quantities are given by

$$
\frac{\rho_{k}}{\rho_{0}}=\frac{N_{k} \bar{n}_{k}}{N_{0} \bar{n}_{0}}, \quad \frac{t_{k}}{t_{0}}=\frac{N_{0}^{2}}{N_{k}^{2}} .
$$

Here, the second relation holds because the typical domain length $\ell(t)$ is by hypothesis proportional both to the inverse total number of domains, and the square root of time. Our purpose will be to determine $\rho_{k}$ and $t_{k}$ as functions of the iteration index $k$, and subsequently by elimination $\rho(t)$.

\subsection{SMAM evolution equation}

The key feature of the SMAM responsible for its analytic solvability is that at every iteration $k$ the number variables $n_{i k}$, for $i=1,2, \ldots, N_{k}$, are uncorrelated. This is because they descend from disjoint sets of 'ancestor' variables. As a result this model obeys an exact closed set of equations, which we shall now derive and analyse. In this subsection we will suppress the iteration index $k$ and will denote, preceding the $k$ th iteration, the number of domains containing precisely $n$ particles by $M_{n}$, the total number of domains by $N=\sum_{n=1}^{\infty} M_{n}$, and the fraction of domains that contain precisely $n$ particles by $f_{n}=M_{n} / N$. For the corresponding quantities after the $k$ th iteration we shall use primed symbols, i.e., $M_{n}^{\prime}, N^{\prime}$, and $f_{n}^{\prime}=M_{n}^{\prime} / N^{\prime}$. By means of appropriate combinatorics one may express $f_{n}^{\prime}$ in terms of the set $\left\{f_{m}\right\}_{m=1}^{\infty}$ before the iteration. We now proceed to derive this relationship by investigating the effect of the successive steps (i) through (iv) of a single iteration.

In step (i) there are $M_{1}=N f_{1}$ domains that become empty. Let $\widetilde{M}_{n}$ be an auxiliary variable indicating the number of domains with $n$ particles (prior to any domain fusion processes), and let $\widetilde{N}=\sum_{n=1}^{\infty} \widetilde{M}_{n}$ denote the total number of domains left after step (ii) of the iteration. Then

$$
\begin{aligned}
& \widetilde{M}_{n}=M_{n+1}=N f_{n+1}, \quad n=1,2,3, \ldots, \\
& \widetilde{N}=N-N_{1}=N\left(1-f_{1}\right) .
\end{aligned}
$$


Next we are interested in the domains that disappear or become created in step (iii). Let $\Delta N_{\text {fusion }}$ denote the net change in the total number of domains due to this process. This number is easily determined as follows: Since the $N f_{1}$ eliminated domains were randomly placed in the lattice, elementary statistics shows that they formed $N_{\mathrm{s}}=N f_{1}\left(1-f_{1}\right)$ sequences of adjacent domains. Their elimination therefore led to $N_{\mathrm{s}}$ new nearest-neighbour pairs. The probability $p_{r}$ for an eliminated sequence to contain exactly $r$ domains is $p_{r}=\left(1-f_{1}\right) f_{1}^{r-1}$, where $r=1,2, \ldots$ It follows that two newly created nearest-neighbour domains / sites will have equal colours with probability

$$
p=\sum_{r=1}^{\infty} p_{r} \eta_{r}=\frac{1}{q-1+f_{1}} .
$$

We remark in passing that whereas in our earlier paper [26] the possibility of $r$ domains disappearing simultaneously was correctly taken into account, the exact expression (23) for $p$ was approximated by its low-density, long-time limiting value $p=1 /(q-1)$. The exact expression (23) used here actually leads to simpler mathematics, and of course does not change the leading power laws; its final consequence is a different amplitude in the subleading term of the end result, Eq. (45).

Since the total number of new nearest-neighbour pairs is $N_{\mathrm{s}}$, the change $\Delta N_{\text {fusion }}$ of the total number of domains due to fusion in step (iii) is

$$
\Delta N_{\text {fusion }}=-N_{\mathrm{s}} p=-N \frac{f_{1}\left(1-f_{1}\right)}{q-1+f_{1}} .
$$

Eqs. (22) and (24) allow us to directly relate $N^{\prime}$ to $N$ according to

$$
N^{\prime}=\widetilde{N}+\Delta N_{\text {fusion }}=N \frac{(q-1)\left(1-f_{1}\right)}{q-1+f_{1}} .
$$

However, we have to study step (iii) in greater detail. For we need to know the numbers $\Delta \widetilde{M}_{n, \text { loss }}$ and $\Delta \widetilde{M}_{n \text {,gain }}$ of domains of size $n$ that are destroyed and created, respectively, during that step. Consider, after step (ii), a specific domain (with arbitrary particle number $n$ ). This very domain will disappear owing to fusion if its left or its right neighbour are of the same colour. The probability $p_{\mathrm{c}}$ that one given neighbour is of the same colour is $p_{\mathrm{c}}=f_{1} /\left(q-1+f_{1}\right)$, namely the product of the probability $f_{1}$ of its originally being adjacent to a group of domains of size 1 , with the probability $1 /\left(q-1+f_{1}\right)$ of this group being adjacent on its other end to a domain of the same colour as the specific one we are considering. Since it has two neighbours, the given domain under consideration will disappear with probability $2 p_{\mathrm{c}}-p_{\mathrm{c}}^{2}$. Since there are altogether $\widetilde{M}_{n}=N f_{n+1}$ domains of size $n$, we find

$$
\begin{aligned}
\Delta \widetilde{M}_{n, \mathrm{loss}} & =-\widetilde{M}_{n}\left(2 p_{\mathrm{c}}-p_{\mathrm{c}}^{2}\right) \\
& =-N f_{n+1}\left[1-\left(\frac{q-1}{q-1+f_{1}}\right)^{2}\right], \quad n=1,2, \ldots
\end{aligned}
$$

Summing this result over $n=1,2, \ldots$ yields the total number $\Delta \tilde{N}_{\text {loss }}$ of domains that 
disappear in step (iii),

$$
\Delta \widetilde{N}_{\text {loss }}=-N\left(1-f_{1}\right)\left[1-\left(\frac{q-1}{q-1+f_{1}}\right)^{2}\right] .
$$

Next we turn to the number $\Delta \widetilde{M}_{n \text {,gain }}$ of $n$-particle domains created in step (iii). Since such a creation may result from the fusion of $s$ domains, we need to determine the corresponding number for each value of $s$, and subsequently sum over all $s=1,2, \ldots$. After step (ii) there are $\widetilde{N}=N\left(1-f_{1}\right)$ domains. We may therefore write

$$
\Delta \widetilde{M}_{n, \text { gain }}=N\left(1-f_{1}\right) \sum_{s=2}^{\infty} x_{s} R_{s n}, \quad n=1,2, \ldots
$$

Here $x_{s}$ represents the probability that a specific domain undergoes a fusion with the $s-1$ domains that follow along the chain, and $R_{s n}$ is the independent probability that this fusion produces a domain of size $n$. We now determine $x_{s}$ and $R_{s n}$. In order that exactly $s$ domains fuse, they must subsequent to step (ii) form a sequence of adjacent domains of the same colour, bordered at both ends by domains of a different colour. The probability for this arrangement is

$$
x_{s}=\left(1-p_{\mathrm{c}}\right)^{2} p_{\mathrm{c}}^{s-1} .
$$

The domain fractions after step (ii) are $\widetilde{f}_{n}=\widetilde{M}_{n} / \widetilde{N}=f_{n+1} /\left(1-f_{1}\right)$, with $n=1,2, \ldots$. The probability that the $s$ fusing domains have sizes $m_{1}, m_{2}, \ldots, m_{s}$ is equal to the product $\widetilde{f}_{m_{1}} \widetilde{f}_{m_{2}} \ldots \widetilde{f}_{m_{s}}$, and the probability that this fusion produces a domain of size $n$ is in turn equal to this product summed on all sizes of the fusing domains, subject to the constraint $m_{1}+m_{2}+\ldots+m_{s}=n$. Hence we have

$$
R_{s n}=\left(1-f_{1}\right)^{-s} \sum_{m_{1}=1}^{\infty} \ldots \sum_{m_{s}=1}^{\infty} f_{m_{1}+1} \ldots f_{m_{s}+1} \delta_{n, m_{1}+\ldots+m_{s}},
$$

Using the preceding expressions in Eq. (28) and summing over $n=1,2, \ldots$ yields

$$
\Delta \widetilde{N}_{\text {gain }}=N \frac{f_{1}\left(1-f_{1}\right)(q-1)}{\left(q-1+f_{1}\right)^{2}} .
$$

From Eqs. (24), (27), and (31) one may check that $\Delta N_{\text {fusion }}=\Delta \widetilde{N}_{\text {gain }}+\Delta \widetilde{N}_{\text {loss }}$, as must of course be the case.

We now consider the recursion

$$
M_{n}^{\prime}=\widetilde{M}_{n}+\Delta \widetilde{M}_{n, \text { loss }}+\Delta \widetilde{M}_{n, \text { gain }}, \quad n=1,2, \ldots,
$$

and divide both sides of this equation by $N^{\prime}$ so that the right-hand side becomes equal to the fraction $f_{n}^{\prime}$. With the aid of several of the preceding equations the right-hand side may then be expressed explicitly in terms of the original fractions $\left\{f_{m}\right\}_{m=1}^{\infty}$. One thereby finds the SMAM evolution equation

$$
f_{n}^{\prime}=\frac{q-1}{\left(1-f_{1}\right)\left(q-1+f_{1}\right)} \mathcal{R}_{n}
$$


valid for $n=1,2, \ldots$, with

$$
\mathcal{R}_{n}=\sum_{s=1}^{\infty} g_{1}^{s-1} \sum_{m_{1}=1}^{\infty} \ldots \sum_{m_{s}=1}^{\infty} f_{m_{1}+1} \ldots f_{m_{s}+1} \delta_{n, m_{1}+\ldots+m_{s}}
$$

and where we have introduced the abbreviation

$$
g_{1}=\frac{f_{1}}{\left(1-f_{1}\right)\left(q-1+f_{1}\right)} .
$$

Eq. (33), together with (34) and (35), constitutes an explicit and fully exact recursion relation for the evolution of the domain fractions under the deterministic SMAM

algorithm. One easily checks that it conserves the sum rule $\sum_{n=1}^{\infty} f_{n}=1$. Recall that the term with index $s$ in the sum in Eq. (34) represents the creation of a domain of $n$ particles by the simultaneous fusion of $s$ domains. The terms with $s \geq 3$ are obviously quite model-specific and one would expect the essential physics to be embodied already in the lowest-order nonlinearity, i.e., in the contribution with $s=2$. However, in spite of the cumbersome appearance of the above recursion, its mathematical analysis turns out to be easier when all terms are retained.

\subsection{Asymptotic density decay}

We now restore the iteration indices $k$ and $k+1$ to replace the unprimed and primed variables, respectively, of the previous subsection. In order to find a solution to the SMAM evolution equation (33)-(35) we substitute as an ansatz an exponential distribution $f_{n k}=\epsilon_{k}\left(1-\epsilon_{k}\right)^{n-1}$ into the right-hand side of Eq. (33). The recursion then reproduces an exponential distribution, $f_{n, k+1}=\epsilon_{k+1}\left(1-\epsilon_{k+1}\right)^{n-1}$, with

$$
\epsilon_{k+1}=\frac{q-1}{q-1+\epsilon_{k}} \epsilon_{k} .
$$

Hence we have indeed identified a solution. For this solution we may express Eq. (25) as

$$
N_{k+1}=\frac{(q-1)\left(1-\epsilon_{k}\right)}{q-1+\epsilon_{k}} N_{k} .
$$

In view of Eq. (21) and the fact that $\bar{n}_{k}=1 / \epsilon_{k}$, dividing Eq. (37) by (36) gives the recursion relation for the particle density $\rho_{k}$,

$$
\rho_{k+1}=\left(1-\epsilon_{k}\right) \rho_{k} .
$$

The random initial distribution that we are considering determines the initial condition for Eq. (36), viz. $\epsilon_{0}=(q-1) / q$, so $f_{n 0}=(q-1) / q^{n}$.

Solving Eqs. (36) and (38) explicitly now appears to be quite simple. From Eq. (36), with the prescribed initial condition, we find

$$
\epsilon_{k}=\frac{q-1}{q+k}
$$

whence, after substitution of this result in Eq. (38),

$$
\frac{\rho_{k}}{\rho_{0}}=\frac{\Gamma(q) \Gamma(k+1)}{\Gamma(k+q)} .
$$


Next we may determine $t_{k}$ with the aid of Eq. (21) and the fact that $\bar{n}_{k}=1 / \epsilon_{k}$,

$$
\frac{t_{k}}{t_{0}}=\left(\frac{\rho_{0} \epsilon_{0}}{\rho_{k} \epsilon_{k}}\right)^{2}=\left[\frac{\Gamma(k+q+1)}{\Gamma(q+1) \Gamma(k+1)}\right]^{2} .
$$

The desired time-dependence of the particle density $\rho(t)$ is then obtained by elimination of the iteration index $k$ from Eqs. (40) and (41) for $\rho_{k}$ and $t_{k}$. We will be able to carry this out explicitly only in an asymptotic expansion for large $k$. For $k \gg q$, one easily finds from Eqs. (40) and (41) that

$$
\begin{aligned}
\frac{\rho_{k}}{\rho_{0}} & =\frac{\Gamma(q)}{k^{q-1}}\left(1-\frac{(q-1) q}{2 k}+\ldots\right), \\
\frac{t_{k}}{t_{0}} & =\frac{k^{2 q}}{[\Gamma(q+1)]^{2}}\left(1+\frac{q(q+1)}{k}+\ldots\right) .
\end{aligned}
$$

Setting $\tau=C t_{k}=[\Gamma(q+1)]^{2} t_{k} / t_{0}$ and inverting the expansion (43) gives

$$
k=\tau^{1 / 2 q}\left(1-\frac{q+1}{2} \tau^{-1 / 2 q}+\ldots\right) .
$$

Finally, substituting Eq. (44) in (42) and identifying $\rho_{k}=\rho\left(t_{k}\right)$ yields

$$
\rho(t) \simeq \rho_{0} \Gamma(q)\left[(C t)^{-(q-1) /(2 q)}+\frac{q-1}{2}(C t)^{-1 / 2}\right],
$$

i.e., the leading-order term of Eq. (45) describes a power-law density decay $\rho(t) \sim t^{-\alpha(q)}$ with the exponent (4); since $\alpha(q)<1 / 2$ we conclude that segregation occurs for all $2 \leq q<\infty$. The correction term in Eq. (45) decays with the power $1 / 2$, which corresponds to the single-species decay law in the absence of segregation. Notice that indeed the relative amplitude of this term grows with $q$. In field theory language, it represents the effect of reaction rate renormalisation (without segregation) due to particle depletion. We note that for large $q$ it will become difficult to numerically distinguish this correction from the leading-order term.

It is not certain that the correction terms that we have identified here for the SMAM have the same relevance for the original MAM as we believe the leading-order term does. Nevertheless, on physical grounds one would expect the presence of a contribution $\sim t^{-1 / 2}$. Indeed, as we shall see in Sec. 4, Monte Carlo simulation estimates of the MAM exponents $\alpha(q)$ in one dimension yield systematically larger values than our prediction (4). We interpret this deviation as the influence of an additional $t^{-1 / 2}$ term, which should also be responsible for the discrepancy between the present theory and earlier simulation results $[25,24]$. In fact, the detailed analysis of their Monte Carlo data, without prior knowledge of our work, led the authors of Ref. [27] to the conclusion that the MAM was best described by a superposition of the two power laws present in Eq. (45).

\subsection{Reaction zone width}

Let $\ell_{\text {int }}$ be the typical interparticle distance between two unequal particles. In one dimension this is also the width of the reaction zone, hypothesised to grow with time 
as $t^{\lambda_{r}(q)}$. Following the arguments of Ref. [21] as exposed in Sec. 1.2, one is directly led to Eq. (9), viz. $\lambda_{r}(q)=(2 q-1) /(4 q)$. For $q=2$ this yields the result $\lambda_{r}(2)=3 / 8$ derived in Ref. [21] for the two-species system. For $q=\infty$ one obtains $\lambda(\infty)=1 / 2$, which means that the reaction zone width grows as fast as the typical domain size. This is possible only if the domains are made up of at most a finite number of particles, in accord with the disappearance of segregation as $q \rightarrow \infty$ and the recovery of $\alpha(\infty)=1 / 2$ as for the single-species system.

\subsection{Connection to the q-state Potts model}

In the continuum limit colliding domain walls coalesce to a single one with probability $(q-2) /(q-1)$ and annihilate with the complementary probability $1 /(q-1)$. This is precisely what happens to domain walls in the dynamics of the one-dimensional zerotemperature $q$-state Potts model. In that context, one may ask for the probability $P_{2}(t)$ that after a time $t$ a domain wall that was initially present has not yet disappeared or coalesced with another domain wall [32]. In the Potts model one sets $P_{2}(t) \sim t^{-\theta(q)}$, with a $q$-dependent persistence exponent $\theta$. Although it is known that $\theta(2)=1 / 2$ and $\theta(\infty)=3 / 2$, so far an explicit expression for the function $\theta(q)$ has not been found (see Ref. [33]).

The same question may be asked for the SMAM. Within this simplified model it is easy to find the answer. The calculation is most easily done in the continuum limit. Here we state only the result, viz. $\theta(q)=1-1 / q$, which is in fact identical to the result obtained by Majumdar and Cornell [34] in a mean-field approximation to the $q$-state Potts model. We feel this agreement is not a coincidence, but due to the fact that the nonfluctuating rate hypothesis at the basis of the SMAM actually represents a kind of mean-field approximation as well. We conjecture that if appropriate fluctuation terms were added to the SMAM evolution equation, then $\alpha(q)$ would remain unchanged, whereas $\theta(q)$ would acquire nontrivial corrections and deviate from its mean-field value.

\section{Monte Carlo simulations}

In this section, we report our Monte Carlo simulation results which aim, within the numerical accuracy at our disposal, to support the above analytical results concerning the exponent $\alpha(q, d)$ in dimensions $d=1,2$, and 3 . We begin with a brief description of our simulation algorithm.

First, as appropriate data structure we chose to implement an array-based pseudobinary tree to minimise the time complexity of the following four procedures: selecting a random entry, adding new entries, removing entries, and retrieving the number of entry matches within the database. In terms of the particle reactions, these processes respectively correspond to selecting a random particle, adding new particles at a given lattice site, removing particles from a given site, and retrieving the number of particles at a given site. This data structure proved to be efficient in single-species reactions. 
Therefore, it was a natural choice to extend its use to multiple particle species. This was accomplished by maintaining separate lists of the particle positions for each species; that is, one instance of the tree data structure would store all $A$ particles, one instance of the tree data structure would store all $B$ particles, etc. As a consequence of this data structure, dropping site exclusion constraints (i.e., allowing only at most one particle per site) requires no extra computational effort, and we also initially allow particles of different species to coexist on the same site. As the simulation process is sufficiently robust to avoid any systematic error in the form of reaction biases, and since by diffusion and reaction processes, the probability of multiple occupancy for any site diminishes rapidly in time, the asymptotic long-time power laws should not be significantly affected by the chosen initial conditions (with the exception of very special correlated initial states in one dimension, as will be discussed below).

Second, the subsequent simulation steps for our implementation of the MAM reactions in $d$ dimensions with $q$ reacting species are as follows:

(i) $q$ (not necessarily empty) sites are selected at random and filled with a particle such that exactly one particle of each species is added to the lattice and only one particle is added to any of the $q$ sites.

(ii) Repeat step (i) until the lattice is filled to the desired initial density, usually 1.

(iii) Increment time by $1 / N(t)$ where $N(t)$ is the total number of particles at this step (i.e., apply an asynchronous time update $[35,11])$.

(iv) Pick a random particle, giving equal weight to all particles; more precisely:

(a) first select the species randomly, weighted by the ratio of the number of particle of the given species to the total particle number;

(b) next, randomly select a particle from this species list, giving equal weight to all particles of this species.

(v) Pick a site randomly from the set of the $2 d$ nearest neighbours of the selected particle, observing periodic boundary conditions.

(vi) If the chosen neighbouring site has no particles of a species different than the selected particle, the selected particle simply hops from its original site to the neighbouring site.

(vii) If the chosen neighbouring site contains particles of a species different than the selected particle, then one of these particles is randomly selected to be removed along with the original particle.

(viii) Repeat steps (iii) through (vii) until no particles are left or until the overall allotted simulation time has elapsed.

In the following, we will present our simulation results for the $q$-species MAM with $q=2,3,4$, and 5. The two-species pair annihilation process $(q=2)$ was investigated numerically rather intensively in one [5, 22], two [5, 36] and three dimensions [21]. For comparison, we will also show the corresponding data for the single-species annihilation process $A+A \rightarrow \varnothing$. As argued before, this process should describe the $q \rightarrow \infty$ limit 


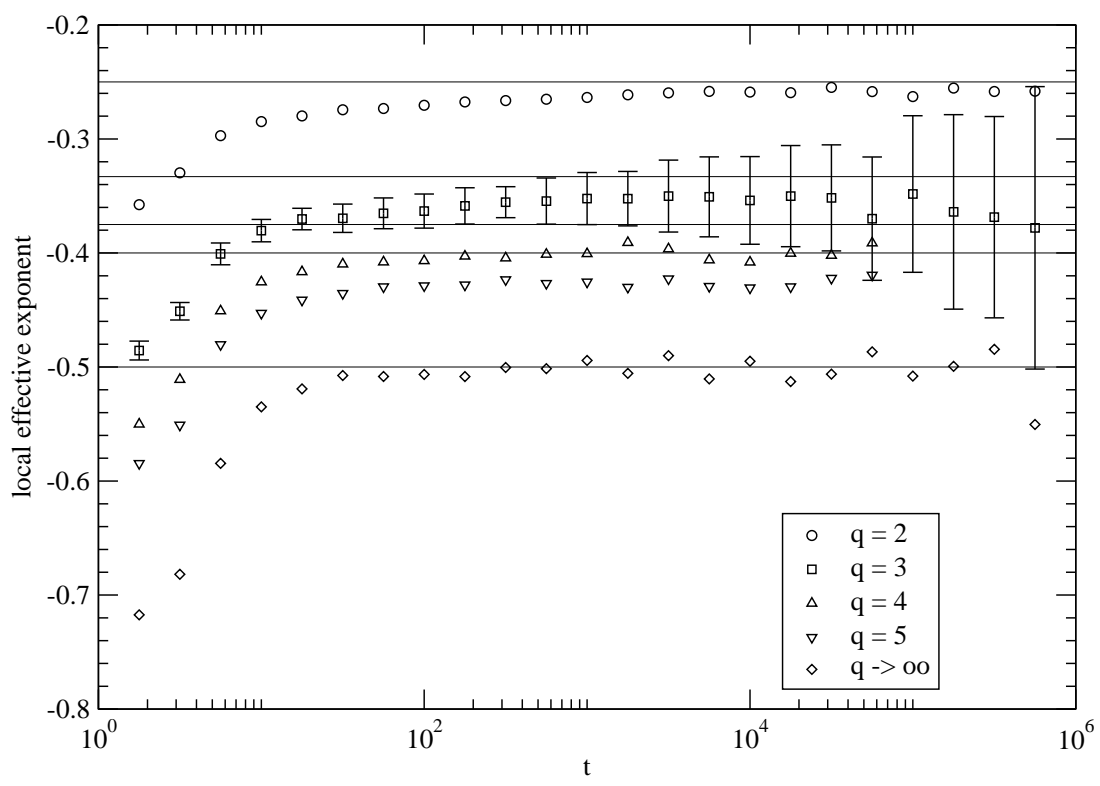

Figure 2. Local effective decay exponent $-\alpha(t)$ for the one-dimensional MAM with $q=2,3,4$, and 5 (random initial conditions, equal initial particle numbers for each species). The data were obtained from $10^{5}$ lattice sites, and are averaged over 50 runs. For the $q=3$ data, we also display the statistical error bars; similar accuracies apply to the other graphs as well. The straight lines correspond to the values $\alpha(q, 1)=(q-1) /(2 q)$ as asymptotically predicted by the SMAM. For comparison, results for the single-species annihilation process $A+A \rightarrow \varnothing$ are displayed also, corresponding to the limit $q \rightarrow \infty$.

of the $q$-species MAM in any dimension. Recently, Zhong, Dawkins, and ben-Avraham have studied the one-dimensional MAM for $q=3,4$, and 5 by means of a renormalised reaction-cell method for systems that are equivalent to lattices with up to $2^{28}$ (!) sites [27].

\subsection{Segregation in the one-dimensional $M A M$}

We begin with the MAM in one dimension, where our previous analysis predicts species segregation to occur for generic (random) initial placements of the particles along the chain. The Monte Carlo simulations were performed on a lattice of $10^{5}$ sites with periodic boundary conditions, and our data sets were averaged over 50 independent runs. For each value of $q$, the long-time density decay is indeed characterised by different exponents $\alpha(q, 1)$, see Refs. [26, 27]. In order to avoid any dependence on the details of the fitting algorithm and also probe the presence of crossover regimes, we follow the standard procedure to measure and plot the 'local' (in time) effective exponent $\alpha(t)$ vs. Monte Carlo time $t$ as defined through

$$
\alpha(t)=-\frac{\mathrm{d} \ln \rho(t)}{\mathrm{d} \ln t},
$$

which in the limit $t \rightarrow \infty$ should approach $\alpha(q, 1)$. 


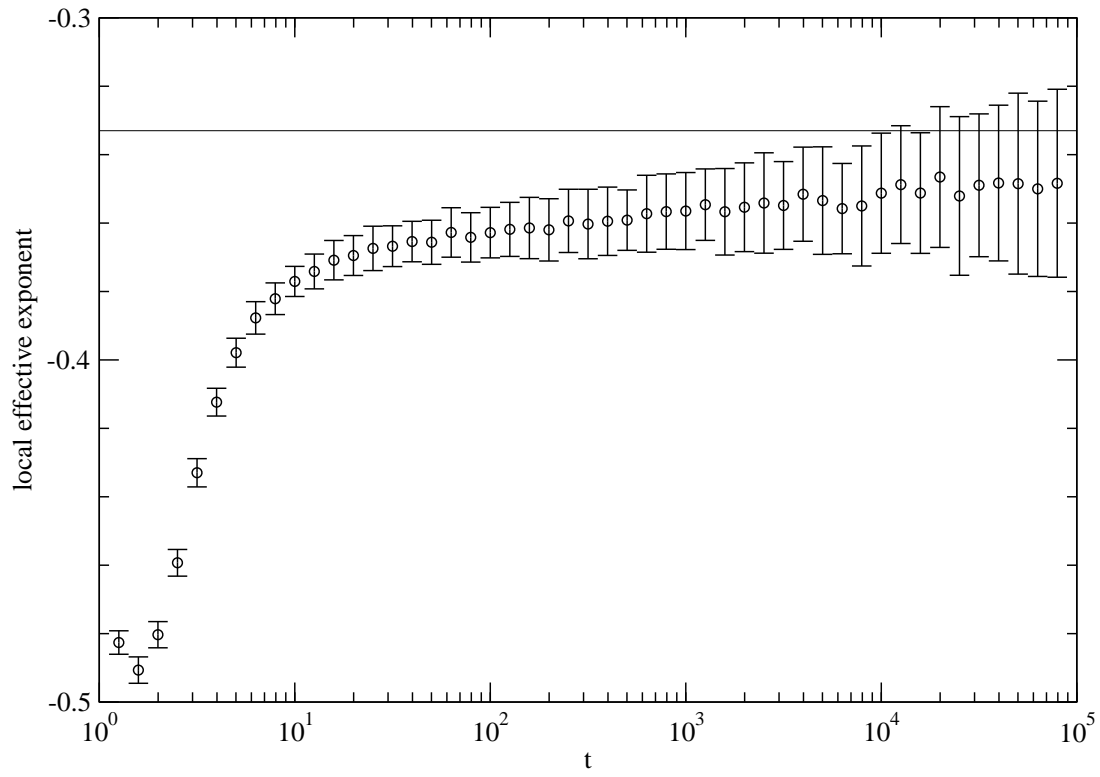

Figure 3. Local effective decay exponent $-\alpha(t)$ for the one-dimensional MAM with three particle species $(q=3)$ with random initial conditions, obtained from averaging over 100 runs on $10^{6}$ lattice sites. The straight line corresponds to the expected value $\alpha(3,1)=1 / 3$.

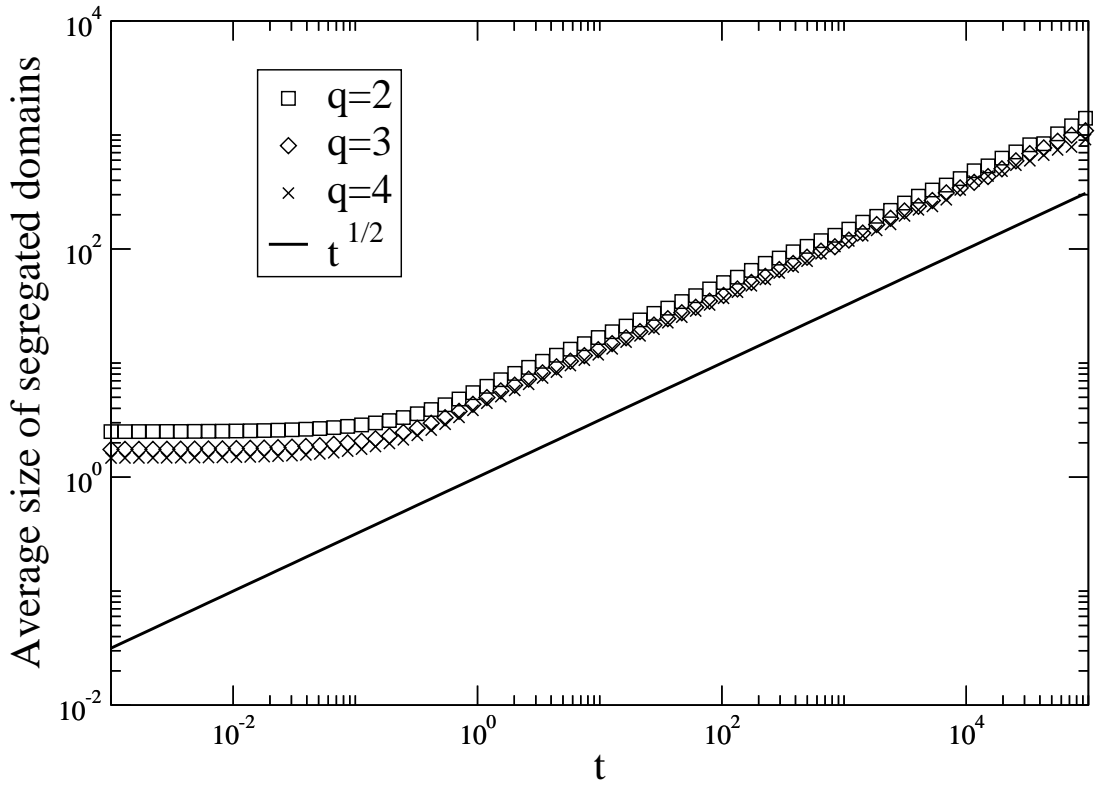

Figure 4. One-dimensional MAM for $q=2,3$, and 4 . The average size of singlespecies segregated regions grows as $t^{1 / 2}$. 
Our data as obtained for the one-dimensional MAM with $q=2,3,4$, and 5 as well as for the single-species pair annihilation (corresponding to the limit $q \rightarrow \infty$ ) are depicted in Fig. 2. The horizontal lines indicate the SMAM predictions $\alpha(2,1)=1 / 4$, $\alpha(3,1)=1 / 3, \alpha(4,1)=3 / 8, \alpha(5,1)=2 / 5$, and $\alpha(\infty, 1)=1 / 2$ (from top to bottom). While the single-species data agree well with the theoretical prediction, for finite $q$ our local effective decay exponent values in the accessible time window are systematically lower than the asymptotic SMAM numbers. We interpret these data to reflect the strong next-to-leading corrections $\sim t^{-1 / 2}$ to the leading density power law decay, $c f$. Eq. (45). This assertion is in fact borne out by the careful data analysis carried out by Zhong et $a l$. for their exceedingly large systems equivalent to lattices of up to $2^{28}$ sites. Yet even then marked corrections to scaling were still prominent. Notice that the simulation data for our system size become rather unreliable for $t>10^{5}$. In Fig. 3 we show simulation results for the three-species MAM $(q=3)$ obtained on a larger lattice with $10^{6}$ sites, averaged over 100 runs. The data appear to systematically but slowly approach the expected decay exponent value $\alpha(3,1)=1 / 3$ at long times.

In addition, we tested a crucial input for our analysis of the SMAM, namely that the size of the segregated domains was assumed to grow with time $\sim t^{1 / 2}$. Neglecting the effect on the asymptotic regime of the single particle domains, one can look at only one realisation, averaging the domain size over all domains in the system to obtain almost noiseless data, as shown in Fig. 4 for $q=2,3$, and 4 . The measured domain growth power law confirms our hypothesis and therefore supports our claim that in the long-time regime the SMAM and the MAM become equivalent.

We also investigated the role of special, highly correlated initial conditions on the long-time MAM evolution. As explained in Sec. 2.5, the separation of distinct species in an ordered alternating alignment ... $A_{q-1} A_{q} A_{1} A_{2} \ldots A_{q-1} A_{q} A_{1} A_{2} \ldots$ will be preserved under the MAM kinetics provided $q$ is even, whence the single-species decay with $\alpha=1 / 2$ should be recovered. For odd $q$, on the other hand, the initial correlations disappear after some crossover period, and eventually segregation will occur, with the density decay governed by the exponents $\alpha(q, 1)$. These predictions are confirmed by our simulation data for $q=2$ (on 65536 sites), $q=3$ (on 98304 sites), and $q=4$ (on 65536 sites), each averaged over 20 runs.

\subsection{Simulation results for the $M A M$ in $d=2$ and $d=3$ dimensions}

For our two-dimensional simulations, we used a $1000 \times 1000$ sites square lattice with periodic boundary conditions in each direction. We have argued above that the singlespecies asymptotic decay law $\rho(t) \sim t^{-1} \ln t$ should apply for all $q \geq 3$ here, in contrast with the two-species process for which $\alpha(2,2)=1 / 2$ as a consequence of the local conservation law for the particle density difference $\rho_{1}-\rho_{2}$. In order to test these assertions, we plot the local effective density decay exponent $\alpha(1)$ for $q=2$, whereas for $q \geq 3$ we define

$$
\bar{\alpha}(t)=-\frac{\mathrm{d} \ln (\rho(t) / \ln t)}{\mathrm{d} \ln t} .
$$




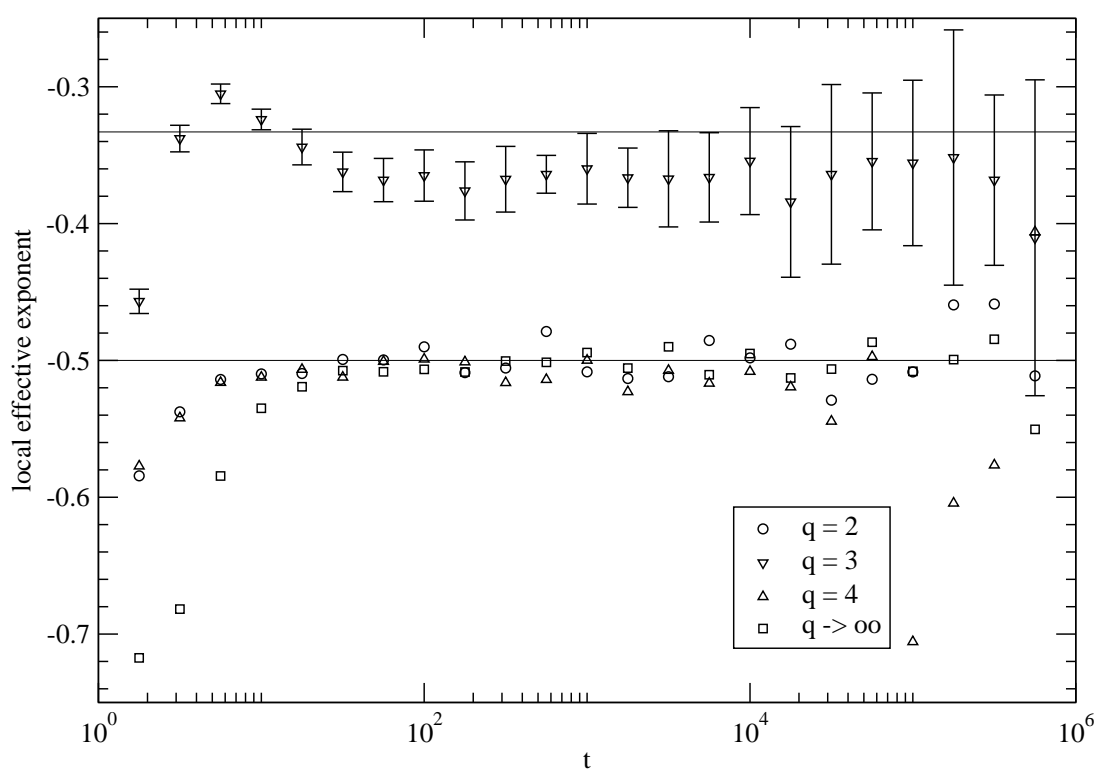

Figure 5. Local effective decay exponent $-\alpha(t)$ for the one-dimensional MAM with alternating initial particle placement for $q=2(\ldots A B A B A B \ldots$ on 65536 sites $), q=3$ (...ABCABC ... on 98304 sites), and $q=4$ (..ABCDABCD . on 65536 sites), each averaged over 20 runs. For the $q=3$ data, we again display the statistical error bars. The straight lines correspond to the theoretical expectations: segregation as for random initial conditions only occurs for odd $q$, while for even $q$ such correlated initial states lead to the single-species decay $\sim t^{-1 / 2}$.

These quantities are plotted in Fig. 6 for $q=2,3,4,5$ and $\infty$ (obtained from the single-species reaction), from data averaged over 50 runs. As expected $\bar{\alpha}(t) \rightarrow 1$ as $t \rightarrow \infty$, which confirms the universal density decay for $d=2$ and hence the absence of species segregation, clearly distinct from the behaviour for $q=2$. We consider the data reliable up to $t=10^{4}$.

Again, the role of correlated initial states can be investigated. To this end, we ran simulations for the two-dimensional two-species annihilation process with checkerboard initial particle placement (alternating ...ABABAB ... rows) on fairly large square lattices with $2048 \times 2048$ sites, averaging the data over 20 runs. In contrast to its onedimensional counterpart, the stochastic reaction kinetics destroys the initial correlations, whence after some crossover period particle segregation emerges and consequently $\rho(t) \sim t^{-1 / 2}$ at long times as for random initial configurations.

In three dimensions, the simulations were performed for $q=2,3,4,5$, and $\infty$ on a $144 \times 144 \times 144$ cubic lattice, and the data averaged over 50 runs. As anticipated above, we observe in Fig. 8 the MAM mean-field behaviour with $\alpha=1$ for $q \geq 3$, which demonstrates again the absence of both reaction rate renormalisation and species segregation in the system. In contrast, the two-species model should still display particle segregation and follow a slower decay with $\alpha(3,2)=3 / 4$. Notice that while our data for $q=2$ clearly show behaviour quite distinct from the cases with $q \geq 3$, our numerical results are markedly off from the expected asymptotic exponent, probably again as a 


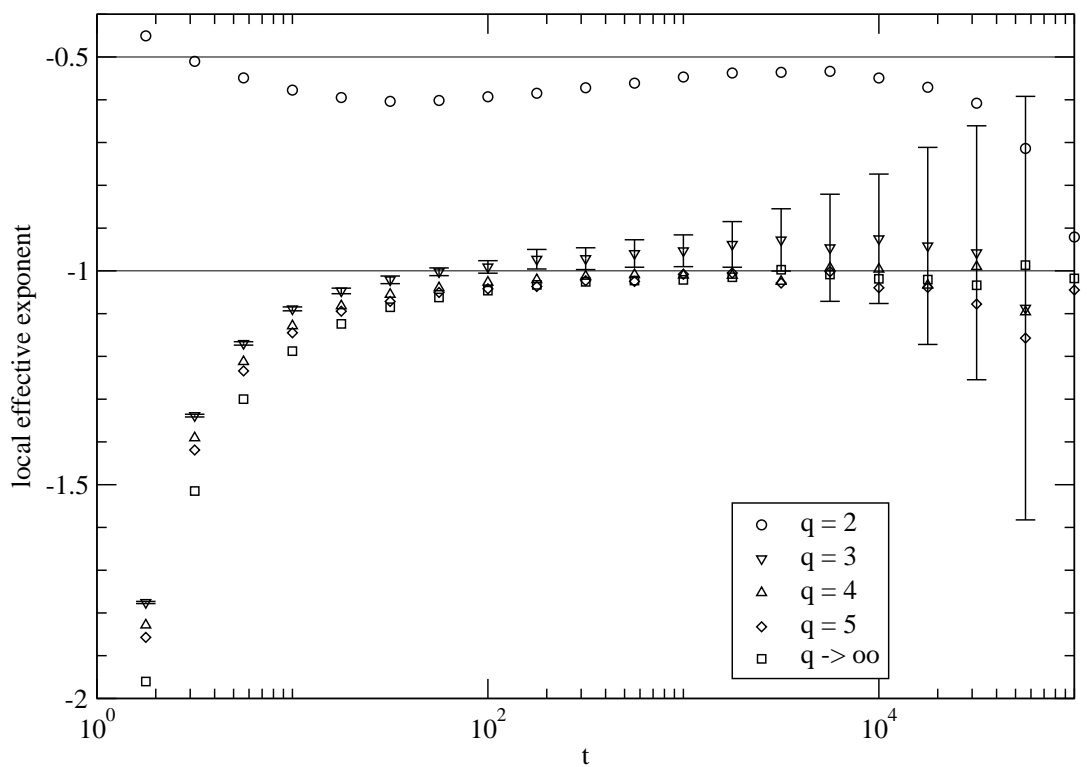

Figure 6. Local effective decay exponent for the MAM in $d=2$ dimensions for $q=2$, 3,4 , and 5 (random initial conditions, equal initial particle numbers for each species). For $q \geq 3$, the effective exponent $\bar{\alpha}(t)$ is obtained from $\rho(t) / \ln t$ rather than the density itself. The data were obtained on a $1000 \times 1000$ square lattice, and are averaged over 50 runs. For the $q=3$ data, we display the statistical error bars. The straight lines correspond to the theoretical expectations, $\rho(t) \sim t^{-1 / 2}$ for the two-species case, and $\rho(t) \sim t^{-1} \ln t$ for $q \geq 3$.

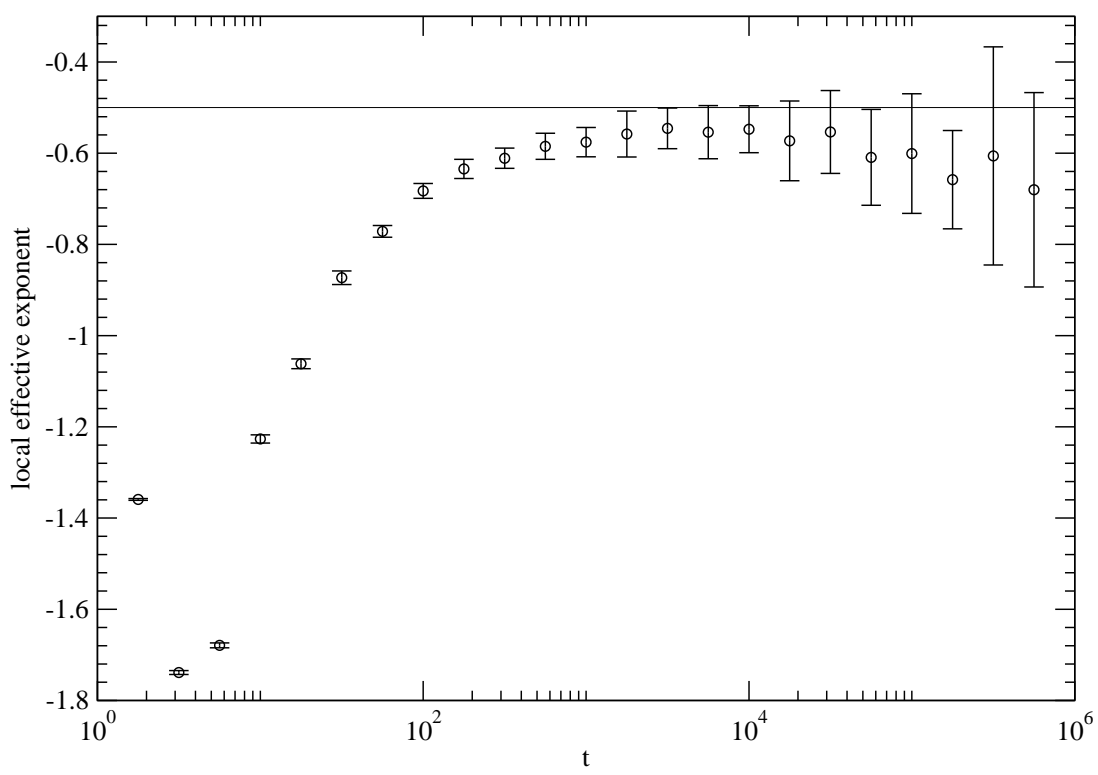

Figure 7. Local effective decay exponent $-\alpha(t)$ for the two-species annihilation process in $d=2$ with checkerboard initial state on $2048 \times 2048$ sites, averaged over 20 runs (with statistical error bars). Following an initial crossover period, this correlated initial state disappears and segregation ensues with $\rho(t) \sim t^{-1 / 2}$ at long times. 


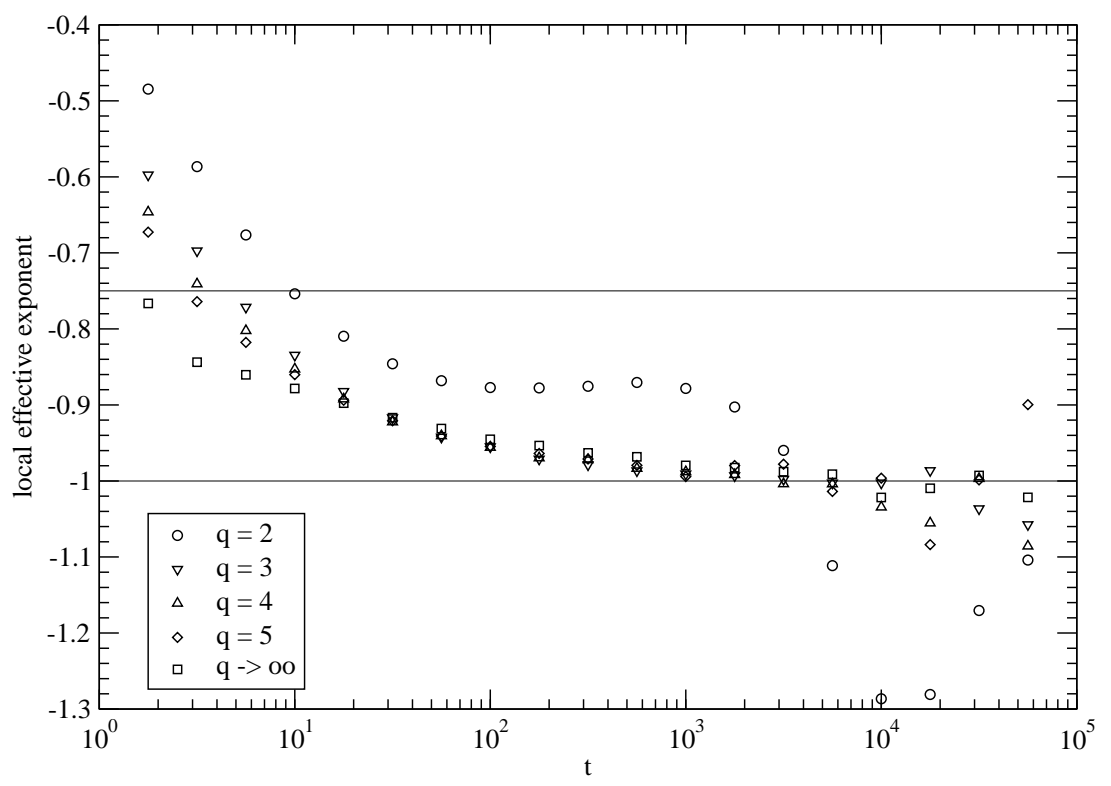

Figure 8. Local effective decay exponent $-\alpha(t)$ for the MAM in $d=3$ dimensions for $q=2,3,4$, and 5 (random initial conditions, equal initial particle numbers for each species). The data were obtained from a $144 \times 144 \times 144$ cubic lattice, and are averaged over 50 runs. The straight lines correspond to the values theoretical expectations, $\rho(t) \sim t^{-3 / 4}$ for the two-species case, and $\rho(t) \sim t^{-1}$ for $q \geq 3$.

consequence of the competing $t^{-1}$ power law. Beyond $t=10^{3}$ the effect of the lattice periodicity sets in and our data are no longer useful for extracting the exponent $\alpha$.

To summarise, our simulation data confirm the universal $q$-independent particle density decay laws for $q \geq 3$ in dimensions $d \geq 2$. It is only due to the presence of an extra and special conservation law that the two-species model behaves qualitatively differently. Correlated initial conditions do not appear to play a crucial role in dimensions $d \geq 2$.

\subsection{Simulation results for two- and three-lane systems}

At last, we were interested in the MAM behaviour on $M$ coupled one-dimensional chains (with periodic boundary conditions along and perpendicular to the chains), which we refer to as $M$-lane systems. Within our systematic and statistical errors, and in the time window accessible to our simulations, our Monte Carlo data for $M=2$ and $M=3$ as depicted in Figs. 9 and 10 yield the same results as for a single one-dimensional chain, perhaps with somewhat different initial crossover regimes. Although particles of different species may now bypass each other, segregation still occurs for $q=2,3$, 4 , and 5 on such few coupled chains, in contrast to the truly two-dimensional system. 


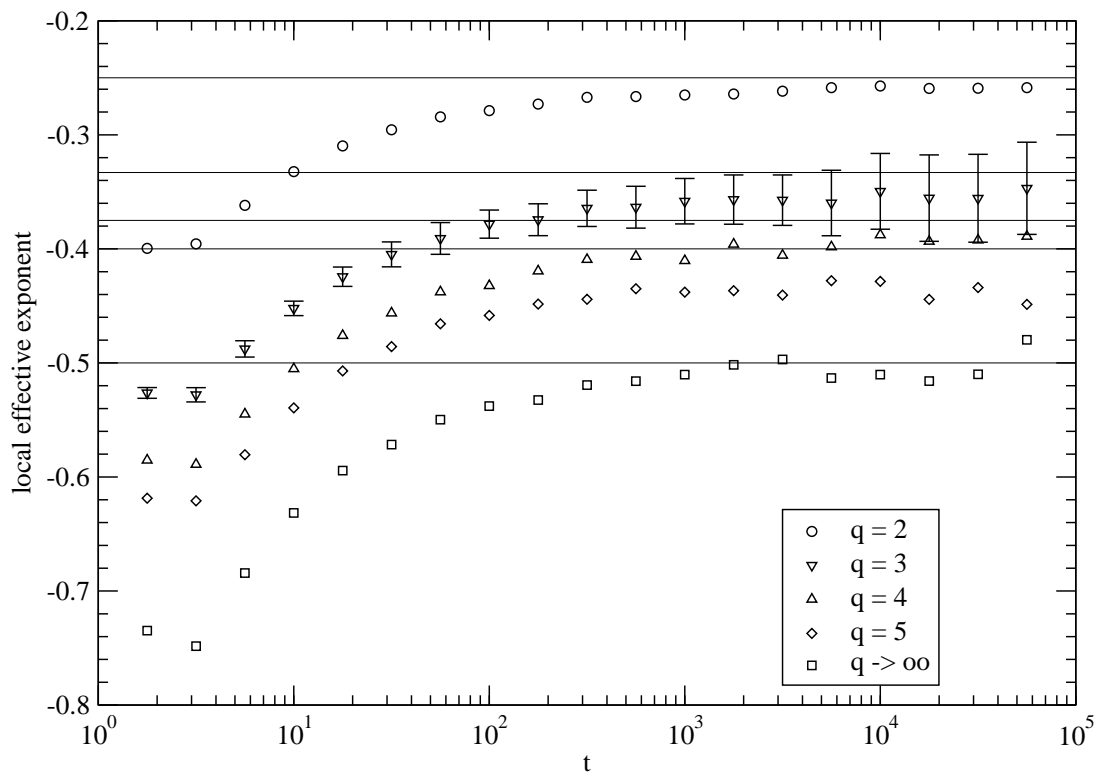

Figure 9. Local effective decay exponent $-\alpha(t)$ for the MAM on a two-lane system for $q=2,3,4$, and 5 (random initial conditions, equal initial particle numbers for each species). The data were obtained on $2 \times 100000$ lattice sites, and are averaged over 50 runs. For the $q=3$ data, we display the statistical error bars. The straight lines correspond to the values $\alpha(q, 1)=(q-1) /(2 q)$ as asymptotically predicted by the one-dimensional SMAM.

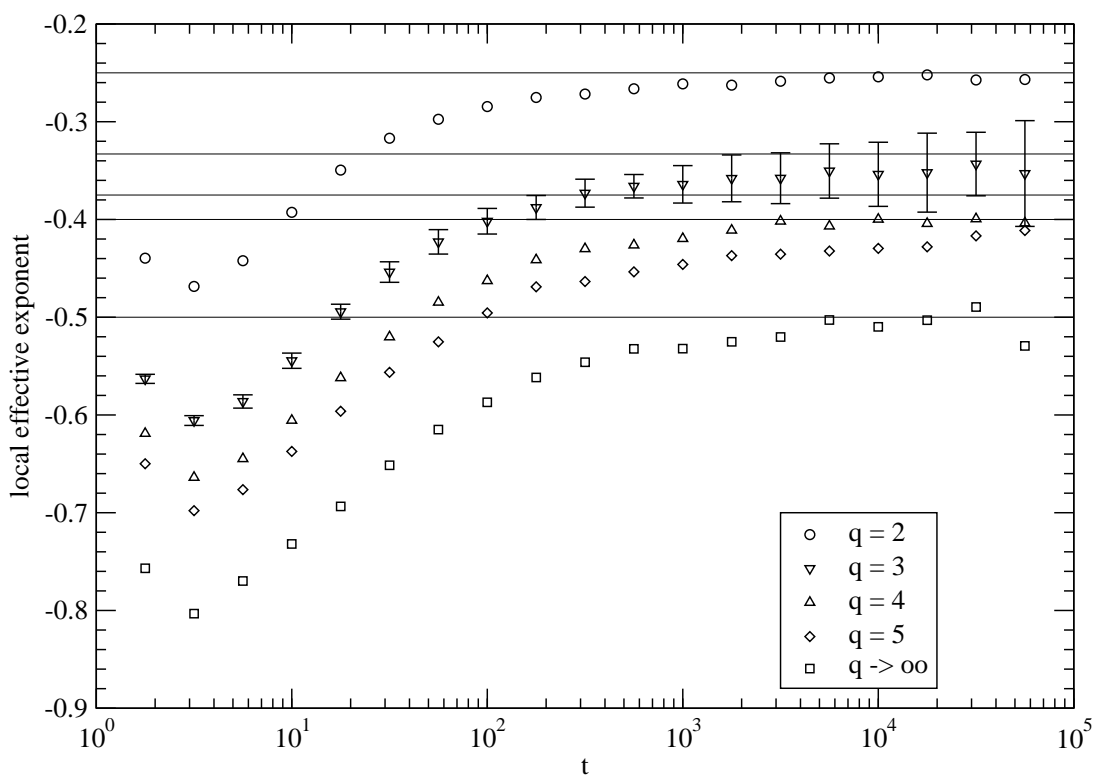

Figure 10. Local effective decay exponent $-\alpha(t)$ for the MAM on a three-lane system for $q=2,3,4$, and 5 (random initial conditions, equal initial particle numbers for each species). The data were obtained on $3 \times 100000$ lattice sites, and are averaged over 50 runs. For the $q=3$ data, we display the statistical error bars. The straight lines correspond to the values $\alpha(q, 1)=(q-1) /(2 q)$ as asymptotically predicted by the one-dimensional SMAM. 


\section{Conclusion}

In this paper we have presented the asymptotic long-time behaviour of the particle density in the $q$-species pair annihilation model in $d$ dimensions. Renormalisation group arguments show that when $q \geq 3$, the total particle density in $d>2$ dimensions decays according to a universal power law $\rho(t) \sim t^{-\alpha}$, with an exponent $\alpha=1$ that is independent of $q$ and $d$. At the upper critical dimension $d_{c}=2$, this mean-field result is supplemented by logarithmic corrections, viz. $\rho(t) \sim t^{-1} \ln t$. In dimensions $d \geq 2$, particle segregation occurs only for the two-species system $(q=2)$, namely for $d<4$, resulting in a slower decay with $\alpha(2, d)=d / 4[4,5,20]$. The case $q=2$ turns out to be quite special owing to the presence of a local conservation law for the difference of the two particle densities. Although consistent with the theoretical predictions, our numerical investigations in $d \geq 2$ could naturally be improved by considering larger systems, longer simulation times, and perhaps a more sophisticated algorithm as developed for the one-dimensional case in Ref. [27].

The topological constraints in one dimension, however, generically induce species segregation, and thereby yields a distinct value for the exponent $\alpha$ for each $q$. A simplified, deterministic cellular automaton version of the model allows us to find $\alpha(q, 1)=(q-1) /(2 q)$. This result has been confirmed numerically through Monte Carlo simulations, which suggests the equivalence between the original stochastic model and the simplified version at least in the accessible time regimes. We have not succeeded in calculating the value of $\alpha(q, 1)$ using field theory methods, which remains an open but difficult task.

It would also be interesting to study different versions of the MAM in more detail [26]. For instance, the $q$-species cyclic annihilation model (q-CAM) with reactions $A_{1}+A_{2} \rightarrow \varnothing, A_{2}+A_{3} \rightarrow \varnothing, \ldots, A_{q}+A_{1} \rightarrow \varnothing$ represents cyclic chemical reactions. For low values of $q$, its properties are easily stated. First of all, the $q$-CAM and $q$-MAM are straightforwardly equivalent when $q=2$ and 3 . Then for $q=4$, species 1 and 3 behave in exactly the same manner, and so do species 2 and 4 . Hence their respective distinction is unnecessary, and the problem is reduced to the 2-MAM, $A+B \rightarrow \varnothing$. In the $q=\infty$ limit, however, the CAM becomes a purely diffusing system with no reaction occurring at all. It is then for $5 \leq q<\infty$ that the $q$-CAM and $q$-MAM can be expected to exhibit different behaviour that poses an intriguing problem for further investigation.

\section{Acknowledgements}

This research has in part been supported by the National Science Foundation through grant nos. DMR-0075725 and DMR-0308548, as well as by the Bank of America Jeffress Memorial Trust, grant no. J-594. We thank Paul Krapivsky for bringing this problem to our attention, and gladly acknowledge helpful discussions with Dani ben-Avraham, John Cardy, Manoj Gopalakrishnan, Sid Redner, Jaime Santos, Beate Schmittmann, Frédéric van Wijland, Ben Vollmayr-Lee, and Royce Zia. 


\section{Appendix A: Solution of the mean-field rate equations (10) and (11)}

We are interested in the solution of the mean-field rate equations (10) with initial conditions that break the permutation symmetry between the $q$ particle species. As shown in the main text, this leads to a system of $p>1$ equations (11) with initial conditions $\rho_{k}(0)=\rho_{k}^{*}$ ordered according to $0<\rho_{1}^{*}<\rho_{2}^{*}<\ldots<\rho_{p}^{*}$, and where the multiplicities $n_{k}$ obviously satisfy the constraint $\sum_{k=1}^{p} n_{k}=q$. (Notice that the case $p=2, q=2$, and hence $n_{1}=n_{2}=1$, corresponds to the process $A+B \rightarrow \varnothing$ with unequal initial densities.) We will now obtain the full solution of this system of ordinary differential equations. Throughout this appendix, asterisks will refer to constants constructed from the initial values. For all $1 \leq k \neq n \leq p$ we have $\rho_{n} \neq \rho_{k}$ and may define

$$
s_{k n} \equiv \frac{\rho_{k}}{\rho_{n}-\rho_{k}}
$$

and from Eq. (11) it follows that these quantities satisfy

$$
\frac{\mathrm{d} s_{k n}(t)}{\mathrm{d} t}=-\rho_{n}(t) s_{k n}(t) .
$$

Specifically, consider the $p-1$ variables $s_{k p}(1 \leq k \leq p-1)$ whose initial values $s_{k p}(0)=s_{k p}^{*} \equiv \rho_{k}^{*} /\left(\rho_{p}^{*}-\rho_{k}^{*}\right)$ are ordered according to

$$
0<s_{1 p}^{*}<s_{2 p}^{*}<\ldots<s_{p-1, p}^{*} .
$$

Eq. (49) shows that the $s_{k p}(t)$ can only decrease with time but must remain positive. Upon dividing two of the equations (49) we find $\mathrm{d} s_{k p} / \mathrm{d} s_{\ell p}=s_{k p} / s_{\ell p}$, which implies that

$$
\frac{s_{k p}}{s_{\ell p}}=\frac{s_{k p}^{*}}{s_{\ell p}^{*}} \equiv c_{k \ell p}^{*}, \quad 1 \leq k, \ell \leq p-1 .
$$

These ratios, of which $p-2$ are independent, constitute constants of the motion of the nonlinear system of ordinary differential equations (11).

Before continuing we briefly elaborate on these constants of the motion. Reasoning as before one easily shows that in fact all ratios $c_{k \ell n} \equiv s_{k n} / s_{\ell n}$ (with $k, \ell, n$ all different) are constants of the motion; but they may be expressed in terms of the $p-2$ already found. There is one more constant of the motion which we do not need explicitly in our present calculation but mention for completeness. It is

$$
C_{n}=\rho_{n}^{q-1} \prod_{\ell=1}^{p} \rho_{\ell}^{-n_{\ell}}\left(\prod_{\substack{\ell=1 \\ \ell \neq n}}^{p} s_{\ell n}\right)^{(q-1) /(p-1)}
$$

where $n$ is arbitrary $\left(C_{m}\right.$ and $C_{n}$ with $m \neq n$ may be expressed through each other with the aid of the $\left.c_{k \ell n}\right)$. Its constancy may be verified by explicit calculation. $C_{n}$ differs from the $c_{k \ell n}$ in that it is proportional to a nonzero power of the densities. For the fully nondegenerate case $\left(p=q\right.$ and hence all $\left.n_{k}=1\right)$ our $C_{1}$ reduces to the constant of the motion found in Ref. [25]. We emphasise, finally, that when one goes beyond the present mean-field approach, these constants of the motion do not correspond to local conservation laws, except in the case of $q=p=2$, where $C_{1}=1 /\left(\rho_{1}-\rho_{2}\right)$. 
As a consequence of Eq. (51), the knowledge of the time evolution of the $s_{k p}$ for a single value of $k$, say $s_{1 p}(t)$, suffices. After dividing Eq. (49) for $k=1$ and $n=p$ by (11) for $k=p$ we obtain

$$
\frac{\mathrm{d} s_{1 p}}{\mathrm{~d} \rho_{p}}=\frac{s_{1 p}}{\sum_{\ell=1}^{p-1} n_{\ell} \rho_{\ell}+\left(n_{p}-1\right) \rho_{p}} .
$$

From Eqs. (48) and (51) we have

$$
\rho_{\ell}=\rho_{p} \frac{s_{1 p}}{s_{1 p}+c_{1 \ell p}^{*}}, \quad \ell=1, \ldots, p-1 .
$$

We next use Eq. (54) in (53) to eliminate $\rho_{1}, \ldots, \rho_{p-1}$ in favour of $\rho_{p}$. This results in an expression for $\mathrm{d} s_{1 p} / \mathrm{d} \rho_{p}$ in terms of $s_{1 p}$ and $\rho_{p}$. Integration of this expression yields $\rho_{p}$ as a function of $s_{1 p}$. Explicitly,

$$
\rho_{p}=\rho_{p}^{*} F\left(s_{1 p}\right)
$$

where

$$
F\left(s_{1 p}\right)=\left(\frac{s_{1 p}}{s_{1 p}^{*}}\right)^{n_{p}-1} \prod_{\ell=1}^{p-1}\left(\frac{s_{1 p}+c_{1 \ell p}^{*}}{s_{1 p}^{*}+c_{1 \ell p}^{*}}\right)^{n_{\ell}} .
$$

Combining (55) with Eq. (49) for $k=1$ and $n=p$ produces the time evolution equation for $s_{1 p}$

$$
\frac{\mathrm{d} s_{1 p}}{\mathrm{~d} t}=-\rho_{p}^{*} s_{1 p} F\left(s_{1 p}\right) .
$$

The full solution is now to be obtained as follows. Solving the differential equation (57) yields $s_{1 p}(t)$, which substituted in (55) yields $\rho_{p}(t)$. These two results together, when used in Eq. (54), produce $\rho_{\ell}(t)$ for all $\ell=1, \ldots, p-1$. For the analysis of (57) it is useful to know that the $c_{1 \ell p}^{*}$ are all positive. We remark parenthetically that they are in fact ordered according to $1=c_{11 p}>c_{12 p}>\ldots>c_{1, p-1, p}>0$.

We will satisfy ourselves here to extract from Eq. (57) the behaviour of $s_{1 p}(t)$ for asymptotically large times. In that limit we may set $s_{1 p}=0$ inside the product, whence Eq. (57) reduces to

$$
\frac{\mathrm{d} s_{1 p}}{\mathrm{~d} t}=-\rho_{p}^{*} A_{n_{p}-1}^{*} s_{1 p}^{n_{p}},
$$

where one may verify without great effort that

$$
A_{n_{p}-1}^{*}=\left(s_{1 p}^{*}\right)^{-n_{p}+1} \prod_{\ell=1}^{p-1}\left(1-\rho_{\ell}^{*} / \rho_{p}^{*}\right)^{n_{\ell}}=\left(s_{1 p}^{*}\right)^{-n_{p}+1} A^{*},
$$

with $A^{*}$ given by Eq. (13). Now two cases have to be distinguished:

Case (i): $n_{p}=1$. This is the situation where the highest density is nondegenerate. Eq. (58) then leads to

$$
s_{1 p}(t) \simeq C^{*} s_{1 p}^{*} \exp \left(-\rho_{p}^{*} A^{*} t\right)
$$

where $C^{*}$ is a constant, and we have factored out $s_{1 p}^{*}$. Combining this result with Eq. (51) we find that in fact

$$
s_{k p}(t) \simeq C^{*} s_{k p}^{*} \exp \left(-\rho_{p}^{*} A^{*} t\right)
$$


for all $k=1, \ldots, p-1$. Upon substituting the asymptotic solution (60) in Eq. (55) we obtain

$$
\rho_{p}(t) \simeq \rho_{p}(\infty)=\rho_{p}^{*} A^{*}
$$

Further analysis shows that the approach is exponential on a time scale $\tau=1 / \rho_{p}^{*} A^{*}$. Finally, from the preceding equation and Eqs. (48) and (61),

$$
\begin{aligned}
\rho_{k}(t) & \simeq C^{*} s_{k p}^{*} \rho_{p}^{*} A^{*} \exp \left(-\rho_{p}^{*} A^{*} t\right) \\
& =C^{*} \frac{\rho_{k}^{*}}{1-\rho_{k}^{*} / \rho_{p}^{*}} A^{*} \exp \left(-\rho_{p}^{*} A^{*} t\right), \quad 1 \leq k \leq p-1 .
\end{aligned}
$$

Conclusion: For $n_{p}=1$ the nondegenerate densest species $\rho_{p}(t)$ tends exponentially to a constant $\rho_{p}^{*} A^{*}$ which depends on all initial densities $\rho_{k}^{*}$ according to Eq. (13). The densities of the other species all tend to zero exponentially. The characteristic time scale is $\tau=1 /\left(\rho_{p}^{*} A_{0}\right)$.

Case (ii): $n_{p}>1$. In this situation the highest density is $n_{p}$-fold degenerate. Eq. (58) now leads to

$$
s_{1 p}(t) \simeq s_{1 p}^{*}\left[\left(n_{p}-1\right) \rho_{p}^{*} A^{*} t\right]^{-1 /\left(n_{p}-1\right)},
$$

where we have again factored out a $s_{1 p}^{*}$. Combining this with Eq. (51) we find that in fact

$$
s_{k p}(t) \simeq s_{k p}^{*}\left[\left(n_{p}-1\right) \rho_{p}^{*} A^{*} t\right]^{-1 /\left(n_{p}-1\right)}
$$

for all $k=1, \ldots, p-1$. Upon substituting the asymptotic solution (64) in Eq. (55) we get

$$
\rho_{p}(t) \simeq \rho_{p}^{*}\left(s_{1 p}(t) / s_{1 p}^{*}\right)^{n_{p}-1} A^{*}=\left[\left(n_{p}-1\right) t\right]^{-1},
$$

which is the mean-field behaviour. Finally, from the preceding equation and Eq. (65),

$$
\rho_{k}(t)=\frac{\rho_{k}^{*}}{\rho_{p}^{*}-\rho_{k}^{*}}\left[\rho_{p}^{*} A^{*}\right]^{-1 /\left(n_{p}-1\right)}\left[\left(n_{p}-1\right) t\right]^{-1-1 /\left(n_{p}-1\right)}, 1 \leq k \leq p-1 .
$$

Conclusion: For $n_{p}>1$ the densest species, which are $n_{p}$-fold degenerate, tend to zero as $t^{-1}$. The other species tend to zero with a faster power, viz. as $t^{-1-1 /\left(n_{p}-1\right)}$.

\section{Appendix B: Mapping onto an antiferromagnetic spin chain in $d=1$}

Our goal is to construct a field theory in which the specificity of the one-dimensional case becomes manifest. To this end, we recall that the single-species annihilation reaction on a lattice can be mapped onto 'quantum' antiferromagnetic $X X Z$ spin chains, provided

we employ site occupation restrictions, i.e., we allow at most a single particle per lattice site $x\left(n_{x}=0\right.$ or 1$)[37,38]$. For annihilation processes that asymptotically approach the empty, absorbing state, this restriction should not matter for the long-time behaviour. Moreover, the $\mathrm{RG}$ fixed point for the renormalised reaction rate in $d<2$ dimensions actually corresponds to an infinite bare annihilation rate, which precludes multiple site occupancy [19]. Instead of using bosonic creation and annihilation operators acting on 
a Hilbert space that allows arbitrarily many particles per site, we define lowering and raising operators $\sigma^{-}$and $\sigma^{+}$that operate on the two possible states on each site,

$$
\sigma^{-}|0\rangle=0, \sigma^{+}|0\rangle=|1\rangle, \sigma^{-}|1\rangle=|0\rangle, \sigma^{+}|1\rangle=0,
$$

and obey the standard anticommutation relations for the spin-1/2 algebra.

For the $q$-MAM, we naturally introduce $q$ operators $\sigma_{x, i}^{ \pm}$which create / destroy a particle of species $i$ on site $x$. It is now a straightforward task to rewrite the classical master equation in the form $\partial_{t}|P(t)\rangle=-\hat{H}|P(t)\rangle$, with a formal state vector $|P(t)\rangle=$ $\sum_{\left\{n_{x, i}=0,1\right\}} P\left(\left\{n_{x, i}\right\}, t\right)\left|\left\{n_{x, i}\right\}\right\rangle$ and the pseudo-Hamiltonian $\hat{H}=\sum_{x, i}\left(\hat{h}_{x, i}^{\mathrm{diff}}+\sum_{j \neq i} \hat{h}_{x, i, j}^{\mathrm{ann}}\right)$, with the diffusion part

$$
\hat{h}_{x, i}^{\text {diff }}=-\frac{D}{2}\left[2 \hat{n}_{x, i}\left(\hat{n}_{x+1, i}-1\right)+\sigma_{x, i}^{+} \sigma_{x+1, i}^{-}+\sigma_{x, i}^{-} \sigma_{x+1, i}^{+}\right],
$$

and the annihilation contribution

$$
\hat{h}_{x, i, j}^{\mathrm{ann}}=\frac{\lambda}{2}\left[\hat{n}_{x, i} \hat{n}_{x+1, j}+\hat{n}_{x, j} \hat{n}_{x+1, i}-\sigma_{x, i}^{-} \sigma_{x+1, j}^{-}-\sigma_{x, j}^{-} \sigma_{x+1, i}^{-}\right],
$$

where we have defined $\hat{n}_{x, i}=\sigma_{x, i}^{+} \sigma_{x, i}^{-}$, and where the summations over $i$ and $j$ extend from 1 to $q$. We remark here that the exclusion constraint is imposed solely on particles of the same species. Since the RG fixed point from the 'bulk' renormalisation of $\lambda$ in the bosonic field theory describes an effectively infinite microscopic reaction rate, we expect that even particles of different species will asymptotically be prevented from occupying identical lattice sites at the same (late) time.

By means of a Jordan-Wigner transformation, one could now proceed to build a fermionic field theory from the spin- $1 / 2$ pseudo-Hamiltonian $\hat{H}$. Indeed, the singlespecies pair annihilation model $A+A \rightarrow \varnothing$ has been analysed in this framework $[39,40,41,42]$. In order to take into account the topological effects specific to the one-dimensional case, we shall follow here the approach of Refs. [43, 44], as described in the Fradkin's textbook [45]. For each spin $\vec{S}$, with quantisation axis oriented along $\vec{n}_{0}$ and eigenstates $\left|S, S_{z}= \pm \hbar / 2\right\rangle$, we introduce a vector $\vec{n}$ of modulus 1 that defines the spin coherent state

$$
|\vec{n}\rangle=\exp \left(-\frac{i \theta}{\hbar} \frac{\vec{n}_{0} \times \vec{n}}{\sin \theta} \cdot \vec{S}\right)\left|S, S_{z}=\hbar / 2\right\rangle, \quad \text { with } \cos \theta=\vec{n}_{0} \cdot \vec{n} .
$$

Each vector $\vec{n}$ has three components (spin coordinates) that we label as $n^{\mu}, n^{\nu}$, and $n^{\rho}$. It can be shown that $\langle\vec{n}|\vec{S}| \vec{n}\rangle=\vec{n} \hbar / 2$, and that for two different vectors $\vec{n}_{1}$ and $\vec{n}_{2}$,

$$
\left\langle\vec{n}_{1} \mid \vec{n}_{2}\right\rangle=\mathrm{e}^{i \Phi\left(\vec{n}_{1}, \vec{n}_{2}, \vec{n}_{0}\right) / 2} \sqrt{\frac{1+\vec{n}_{1} \cdot \vec{n}_{2}}{2}}
$$

where $\Phi\left(\vec{n}_{1}, \vec{n}_{2}, \vec{n}_{0}\right)$ represents the oriented surface area of the triangle on the unit sphere whose vertices are given by the endpoints of the three vectors $\vec{n}_{1}, \vec{n}_{2}$, and (fixed) $\vec{n}_{0}$.

Through manipulation of these coherent states, the average of an observable can be written as in Eq. (16). In discrete time (with the time interval $\left[t_{0}, t_{f}\right]$ divided into $N=\left(t_{f}-t_{0}\right) / \delta t$ time steps, the limit $\delta t \rightarrow 0$ to be taken in the end) and on the lattice, 
the ensuing action takes the form

$$
\begin{aligned}
\mathcal{S}[\vec{n}]= & -\frac{i}{2} \sum_{x=1}^{L} \sum_{i=1}^{q} \sum_{k=1}^{N}\left\{\Phi\left(\vec{n}_{x, i}\left(t_{k}\right), \vec{n}_{x, i}\left(t_{k-1}\right), \vec{n}_{0}\right)\right. \\
& \left.-\frac{1}{2} \ln \frac{1+\vec{n}_{x, i}\left(t_{k}\right) \cdot \vec{n}_{x, i}\left(t_{k+1}\right)}{2}+\delta t\left\langle\vec{n}_{x, i}\left(t_{k}\right)|\hat{H}| \vec{n}_{x, i}\left(t_{k}\right)\right\rangle\right\},
\end{aligned}
$$

Here, the first contribution (with $\delta t \rightarrow 0$ ) is of topological origin, and usually called Wess-Zumino term [45]. It is solely responsible for the differences between quantum ferromagnets and antiferromagnets, as well as for the remarkable qualitative distinction that emerges between half-integer and integer antiferromagnetic spin chains. But notice its nonlocal character, since it represents an oriented surface area, yielding disjoint sectors each characterised by a certain value of the corresponding topological charge. This renders a field theory analysis in terms of a local perturbative expansion futile.

Let us briefly discuss the single-species pair annihilation model $A+A \rightarrow \varnothing$ (which also describes the $q=\infty$ limit of the $q$-MAM) in this framework. One obtains explicitly

$$
\begin{aligned}
& \left\langle\vec{n}_{x}\left(t_{k}\right)|\hat{H}| \vec{n}_{x}\left(t_{k}\right)\right\rangle=\frac{\lambda-D}{4} \vec{n}_{x}\left(t_{k}\right) \cdot \vec{n}_{x+1}\left(t_{k}\right) \\
& +\frac{\lambda}{4}\left[2 n_{x}^{\rho}\left(t_{k}\right)-2 n_{x}^{\mu}\left(t_{k}\right) n_{x+1}^{\mu}\left(t_{k}\right)+i n_{x}^{\mu}\left(t_{k}\right) n_{x+1}^{\nu}\left(t_{k}\right)+i n_{x}^{\nu}\left(t_{k}\right) n_{x+1}^{\mu}\left(t_{k}\right)\right] .
\end{aligned}
$$

In one dimension, the reactions will be diffusion-limited, i.e., the ratio of the annihilation and diffusion rate $\lambda / D>1$. Consequently, the isotropic Heisenberg spin coupling in the first term is antiferromagnetic (in contrast to a reaction-limited situation, or to a purely diffusive model) [39, 40, 41]. Following Ref. [45], we therefore assume that the ground state is represented via the ansatz $\vec{n}_{x}=(-1)^{x} \vec{m}_{x}+a_{0} \vec{\ell}_{x}$, where $a_{0}$ denotes the lattice constant. With this change of variables, we may now study the field fluctuations around the antiferromagnetic ground state. We now take the continuum limit $\left(a_{0} \rightarrow 0\right.$ and $\delta t \rightarrow 0)$, and integrate out the field $\vec{\ell}$, whereupon after straightforward manipulations we arrive at the effective action $S=S_{0}+S_{\text {int }}+S_{\text {top }}$, with the harmonic part

$$
\begin{aligned}
S_{0} & =\int \mathrm{d} x \int \mathrm{d} t\left[\frac{\lambda}{a_{0}} m^{\mu 2}-\frac{i \lambda}{a_{0}} m^{\mu} m^{\nu}+\frac{i \lambda}{4 a_{0}(\lambda-2 D)}\left(m^{\mu} \partial_{t} m^{\nu}-m^{\nu} \partial_{t} m^{\mu}\right)\right. \\
& \left.-\frac{a_{0}(\lambda+2 D)}{4}\left(\partial_{x} m^{\mu}\right)^{2}+\frac{a_{0}(\lambda-2 D)}{4}\left[\left(\partial_{x} m^{\nu}\right)^{2}+\left(\partial_{x} m^{\rho}\right)^{2}\right]+\frac{i \lambda a_{0}}{2}\left(\partial_{x} m^{\mu}\right)\left(\partial_{x} m^{\nu}\right)\right]
\end{aligned}
$$

and the nonlinear contributions

$$
\begin{aligned}
S_{\text {int }}= & \int \mathrm{d} x \int \mathrm{d} t\left[\frac{1}{16 a_{0}(\lambda-2 D)}\left(m^{\mu} \partial_{t} m^{\nu}-m^{\nu} \partial_{t} m^{\mu}\right)^{2}\right. \\
& +\frac{\lambda-2 D}{4 a_{0}(\lambda-4 D)^{2}}\left(m^{\nu} \partial_{t} m^{\rho}-m^{\rho} \partial_{t} m^{\nu}\right)^{2}-\frac{D}{2 a_{0}(\lambda-4 D)^{2}}\left(m^{\rho} \partial_{t} m^{\mu}-m^{\mu} \partial_{t} m^{\rho}\right)^{2} \\
& \left.\quad-\frac{i \lambda}{4 a_{0}(\lambda-4 D)^{2}}\left(m^{\nu} \partial_{t} m^{\rho}-m^{\rho} \partial_{t} m^{\nu}\right)\left(m^{\rho} \partial_{t} m^{\mu}-m^{\mu} \partial_{t} m^{\rho}\right)\right]
\end{aligned}
$$

The topological term reads as in Eq. (73), with $q=1$. We have furthermore omitted the ln contribution, since it turns out to be irrelevant. The particle kinetics or, equivalently, the spin dynamics can now be examined by diagonalising the quadratic part of the action 
$S_{0}$. To this end, we Fourier-transform Eq. (75) and then determine the eigenvalues of the ensuing coupling matrix. Let $k$ and $\omega$ be the Fourier variables in space and time, respectively. We then find that the modes along $m^{\rho}$ are static. In the $\left(m^{\mu}, m^{\nu}\right)$ plane, however, there exist propagating wave solutions with the long-wavelength and low-energy dispersion

$$
q^{2} \approx \frac{\lambda}{D a_{0}^{2}}\left(1 \pm \frac{i \omega}{\lambda-2 D}\right) .
$$

Whereas the particle propagation is purely diffusive for $\lambda=0$, the presence of the annihilation reactions $(\lambda>0)$ produces an exponential decay of the propagator amplitude. Unlike the equivalent bosonic field theory, the full action given by Eqs. (75) and (76) is not easily analysed. As in the theory of equilibrium quantum spin chains, other methods of analysis such as the Bethe ansatz have proven more fruitful to extract the long-time behaviour of such systems.

We now return to the spin representation of the multi-species annihilation model. As evident from Eq. (73), each particle species now carries its own topological charge, described by the corresponding Wess-Zumino term. Whereas the diffusion part is still diagonal in the species index,

$$
\left\langle\vec{n}_{x, i}\left(t_{k}\right)|\hat{H}| \vec{n}_{x, i}\left(t_{k}\right)\right\rangle^{\mathrm{diff}}=-\frac{D}{4} \vec{n}_{x, i}\left(t_{k}\right) \cdot \vec{n}_{x+1, i}\left(t_{k}\right),
$$

the annihilation reactions now couple distinct particle species,

$$
\begin{gathered}
\left\langle\vec{n}_{x, i}\left(t_{k}\right)|\hat{H}| \vec{n}_{x, i}\left(t_{k}\right)\right\rangle^{\mathrm{ann}}=\frac{\lambda}{4} \sum_{j \neq i}\left[\vec{n}_{x, i}\left(t_{k}\right) \cdot \vec{n}_{x+1, j}\left(t_{k}\right)+n_{x, i}^{\rho}\left(t_{k}\right)+n_{x, j}^{\rho}\left(t_{k}\right)\right. \\
\left.-2 n_{x, i}^{\mu}\left(t_{k}\right) n_{x+1, j}^{\mu}\left(t_{k}\right)+i n_{x, i}^{\mu}\left(t_{k}\right) n_{x+1, j}^{\nu}\left(t_{k}\right)+i n_{x, i}^{\nu}\left(t_{k}\right) n_{x+1, j}^{\mu}\left(t_{k}\right)\right]
\end{gathered}
$$

and thus in effect link different topological sectors. This fact further exacerbates the difficulties with the spin representation of the $q$-MAM, and we consider it rather unlikely that this formalism will permit a reliable determination of the decay exponent $\alpha(q)$. But the presence of the nonlocal topological terms explains why, for integer $q$ at least, the $q$-MAM displays quite different behaviour in $d=1$ as compared to higher dimensions. For, since we expect the ground state to be characterised by Néel antiferromagnetic order, already in two dimensions the contributions of adjacent chains should cancel the effects of the Wess-Zumino terms at least at sufficiently long wavelengths [45, 46]. For integer $q$ therefore, one would expect special properties, and a dependence of the asymptotic scaling regime on the value of $q$, only in one dimension.

\section{References}

[1] See, e.g., B. Chopard and M. Droz, Cellular Automaton Modeling of Physical Systems (Cambridge University Press, Cambridge 1998).

[2] J. Marro and R. Dickman, Nonequilibrium Phase Transitions in Lattice Models (Cambridge University Press, Cambridge 1999).

[3] M. Smoluchowski, Physik Z. 17, 557 (1916).

[4] A. A. Ovchinnikov and Y. B. Zeldovich, Chem. Phys. 28, 215 (1978). 
[5] D. Toussaint and F. Wilczek, J. Chem. Phys. 78, 2642 (1983).

[6] V. Kuzovkov and E. Kotomin, Rep. Prog. Phys. 51, 1479 (1988).

[7] A. A. Ovchinnikov, S. F. Timashev, and A. A. Belyy, Kinetics of Diffusion-Controlled Chemical Processes (Nova Science, New York, 1989).

[8] W. Dieterich, P. Fulde, and I. Peschel Adv. Phys. 29, 527 (1980).

[9] D. Molisson, J. Roy. Stat. Soc. B 39, 283 (1977).

[10] E. V. Albano, J. Phys. A 27, L881 (1994).

[11] H. Hinrichsen, Adv. Phys. 49, 815 (2000).

[12] G. Ódor, e-print cond-mat/0205644.

[13] L. Peliti, J. Phys. A 19, L365 (1986).

[14] R. Kroon, H. Fleurent, and R. Sprik, Phys. Rev. E 47, 2462 (1993).

[15] M. Bramson and D. Griffeath, Ann. Prob. 8, 183 (1980).

[16] M. Doi, J. Phys. A 9, 1465 (1976).

[17] P. Grassberger and P. Scheunert, Fortschr. Phys. 28, 547 (1980).

[18] L. Peliti, J. Phys. (Paris) 46, 1469 (1985).

[19] B. P. Lee, J. Phys. A 27, 2633 (1994).

[20] B. P. Lee and J. Cardy, J. Stat. Phys. 80, 971 (1995).

[21] F. Leyvraz and S. Redner, Phys. Rev. A 46, 3132 (1992).

[22] K. Kang and S. Redner, Phys. Rev. Lett. 52, 955 (1984).

[23] M. Bramson and J. L. Lebowitz, Phys. Rev. Lett. 61, 2397 (1988); J. Stat. Phys. 65, 941 (1991).

[24] P. Krapivsky, private communication (2000).

[25] D. Ben-Avraham and S. Redner, Phys. Rev. A 34, 501 (1986).

[26] O. Deloubrière, H. J. Hilhorst, and U. C. Täuber, Phys. Rev. Lett. 89, 250601 (2002).

[27] D. Zhong, R. Dawkins, and D. ben-Avraham, Phys. Rev. E 67, 040101 (2003).

[28] E. Ben-Naim and P. L. Krapivsky, e-print cond-mat/0312509.

[29] For a recent overview, see: G. Ódor and N. Menyhárd, Physica D 168, 305 (2002).

[30] K. Oerding, J. Phys. A 29, 7051 (1996).

[31] B. P. Lee and J. Cardy, J. Stat. Phys. 87, 951 (1997).

[32] This question was brought to our attention by S. Majumdar.

[33] S. J. O'Donoghue and A. J. Bray, Phys. Rev. E 65, 051113; 051114 (2002).

[34] S. Majumdar and S. Cornell, Phys. Rev. E 57, 3757 (1998).

[35] V. Privman, Ed., Nonequilibrium statistical mechanics in one dimension, Cambridge University Press (1997).

[36] S. Cornell, M. Droz, and B. Chopard, Physica A 188, 322 (1992).

[37] F.C. Alcaraz, M. Droz, M. Henkel, and V. Rittenberg, Ann. Phys. (NY) 230, 250 (1994).

[38] M. Henkel, E. Orlandini, and J. Santos, Ann. Phys. (NY) 259, 163 (1997).

[39] G. M. Schütz, J. Phys. A 28, 3405 (1995).

[40] G. M. Schütz, Phys. Rev. E 53, 1475 (1996).

[41] J. E. Santos, G. M. Schütz, and R. B. Stinchcombe, J. Chem. Phys 105 (6), 2399 (1996).

[42] V. Brunel, K. Oerding, and F. van Wijland J. Phys. A 33, 1085 (2000).

[43] P. Wiegmann, Phys. Rev. Lett. 60, 821 (1988).

[44] E. Fradkin and M. Stone, Phys. Rev. B 38, 7215 (1988).

[45] E. Fradkin, Field Theories of Condensed Matter Systems Frontiers in physics, (Perseus books publishing, Massachusetts, 1991).

[46] Recall, however, that our simulation data for the MAM in two- and three-lane systems markedly display the one-dimensional power laws associated with species segregation. 